\title{
14-Bit Fully Differential SAR ADC with PGA Used in Readout Circuit of CMOS Image Sensor
}

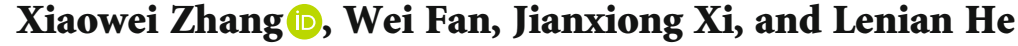 \\ Institute of VLSI Design, Zhejiang University, Hangzhou 310027, China \\ Correspondence should be addressed to Lenian He; helenian@zju.edu.cn
}

Received 13 October 2020; Revised 23 January 2021; Accepted 27 January 2021; Published 23 February 2021

Academic Editor: Changhui $\mathrm{Hu}$

Copyright ( 2021 Xiaowei Zhang et al. This is an open access article distributed under the Creative Commons Attribution License, which permits unrestricted use, distribution, and reproduction in any medium, provided the original work is properly cited.

\begin{abstract}
This paper proposes a 14-bit fully differential Successive Approximation Register (SAR) Analog-to-Digital Converter (ADC) with a programmable gain amplifier (PGA) used in the readout circuit of CMOS image sensor (CIS). SAR ADC adopts two-step scaledreference voltages to realize 14-bit conversion, aimed at reducing the scale of capacitor array and avoiding using calibration to mitigate the impact of offset and mismatch. However, the reference voltage self-calibration algorithm is applied on the design to guarantee the precision of reference voltages, which affects the results of conversion. The three-way PGA provides three types of gains: $3 \mathrm{x}, 4 \mathrm{x}$, and $6 \mathrm{x}$, and samples at the same time to get three columns of pixel signal and increase the system speed. The pixel array of the mentioned CIS is $1026 \times 1024$, and the pixel pitch is $12.5 \mu \mathrm{m} \times 12.5 \mu \mathrm{m}$. The prototype chip is fabricated in the $180 \mathrm{~nm}$ CMOS process, and both digital and analog voltages are $3.3 \mathrm{~V}$. The total area of the chip is $6.25 \times 18.38 \mathrm{~mm}^{2}$. At $150 \mathrm{kS} / \mathrm{s}$ sampling rate, the SNR of SAR ADC is $71.72 \mathrm{~dB}$ and the SFDR is $82.91 \mathrm{~dB}$. What is more, the single SAR ADC consumes $477.2 \mathrm{uW}$ with the $4.8 V_{\mathrm{PP}}$ differential input signal and the total power consumption of the CIS is about $613 \mathrm{~mW}$.
\end{abstract}

\section{Introduction}

An image sensor is a device that converts light signals into electrical signals. In recent years, the demand for an image sensor is continuously increasing, which is widely used in mobile phones, SLR digital cameras, automotive electronics, and security industry fields [1]. Mainstream image sensor technologies are roughly divided into two types: CCD image sensor and CMOS image sensor (CIS). With the advantages of high resolution and low noise, CIS gradually becomes the first choice of sophisticated and important fields. Readout circuit is the core part of CIS, whose continuous speed is normally between $50 \mathrm{fps}$ and $10 \mathrm{Mfps}$ while ADC is the important module in readout circuit $[2,3]$. Based on the number of ADCs used in a circuit, column ADC may be the most suitable ADC applied in the large pixel array, keeping a good balance between area, power, and speed while the other types are chip ADC and pixel ADC. With the size of pixel array increasing, the mutual interference between signals will increase the complexity of the system when the pixel array studied in most researches is less than $1000 \mathrm{H} * 1000 \mathrm{~V}$ [4-
6]. Combined with the above situation, SAR ADC is employed widely when the scale of pixel array increases. Compared with other types of ADC, the balance between power consumption and speed is always the advantage of SAR ADC. In order to maximize the dynamic range of SAR $\mathrm{ADC}$, a programmable gain amplifier (PGA) is the necessary part used in weak-light conditions, though it usually consumes a substantial amount of power. The performance of SAR ADC and PGA directly affects the quality of the images captured by the image sensor while the bits of SAR ADC decide the resolution and the PGA determines the dynamic range.

The pixel array of CIS presented in this paper is $1026 \times$ 1024 , and the pixel size is $12.5 \mu \mathrm{m} \times 12.5 \mu \mathrm{m}$, which is larger than normal CIS. In order to read signal as fast as possible, column readout circuit is adopted. The resolution of SAR ADC is 14 bits, which is relatively high. Usually, a calibration algorithm is adopted in this situation while the SAR ADC used in this paper do not follow the mainstream practice, considering the power, area, and complexity. However, a reference voltage precision optimization algorithm is used to 


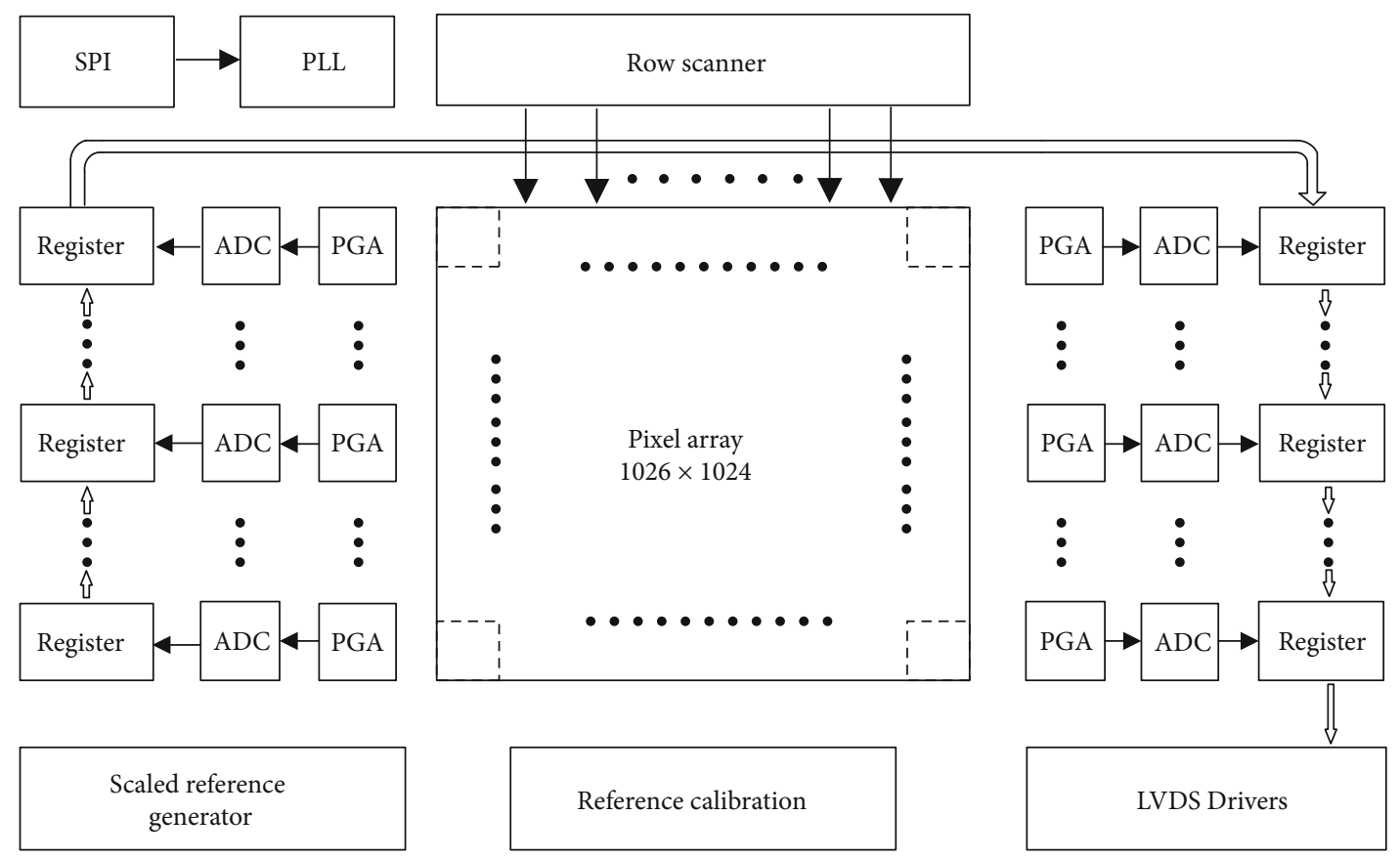

FIGURE 1: System architecture of the proposed CIS.

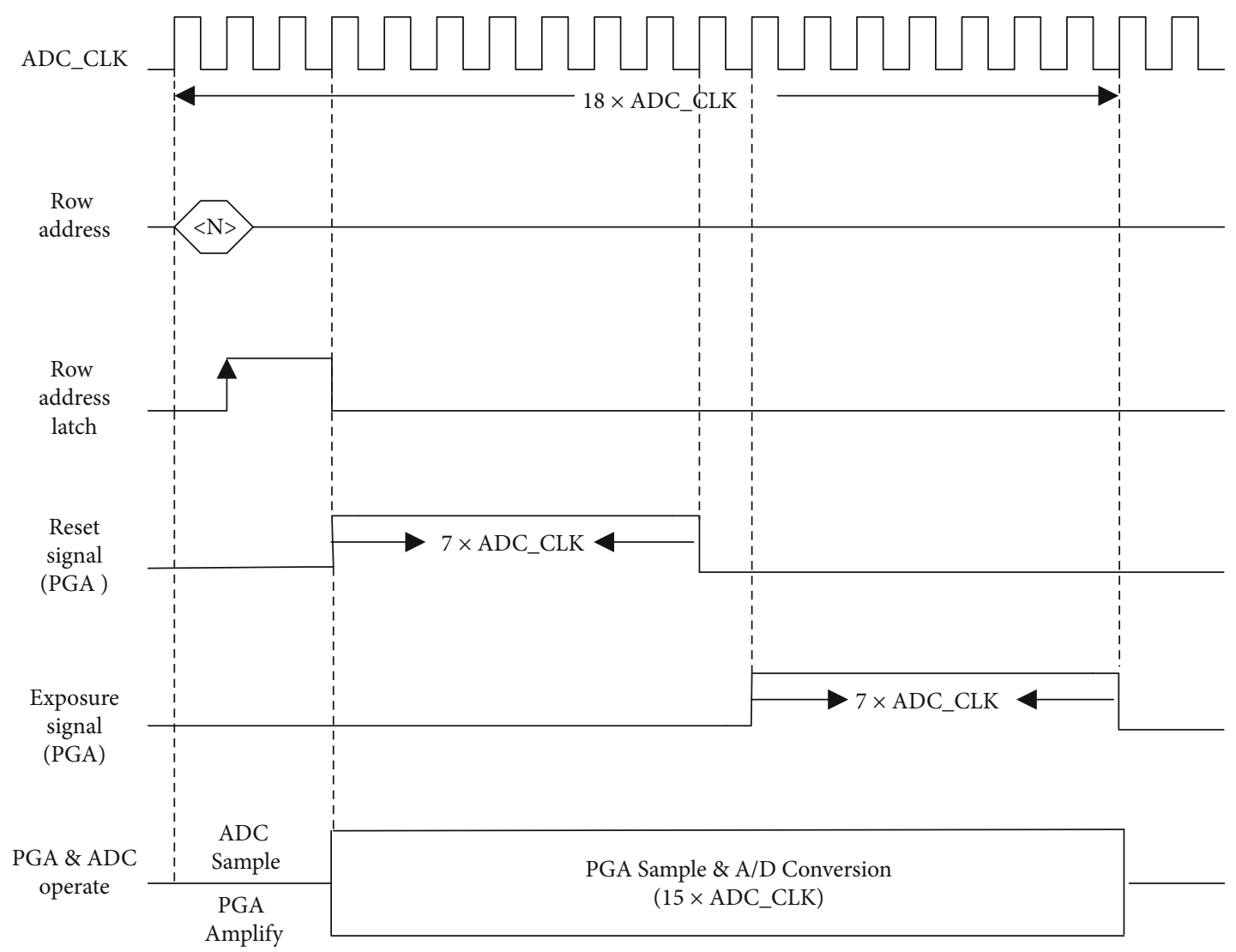

FIGURE 2: System timing of the proposed CIS. 


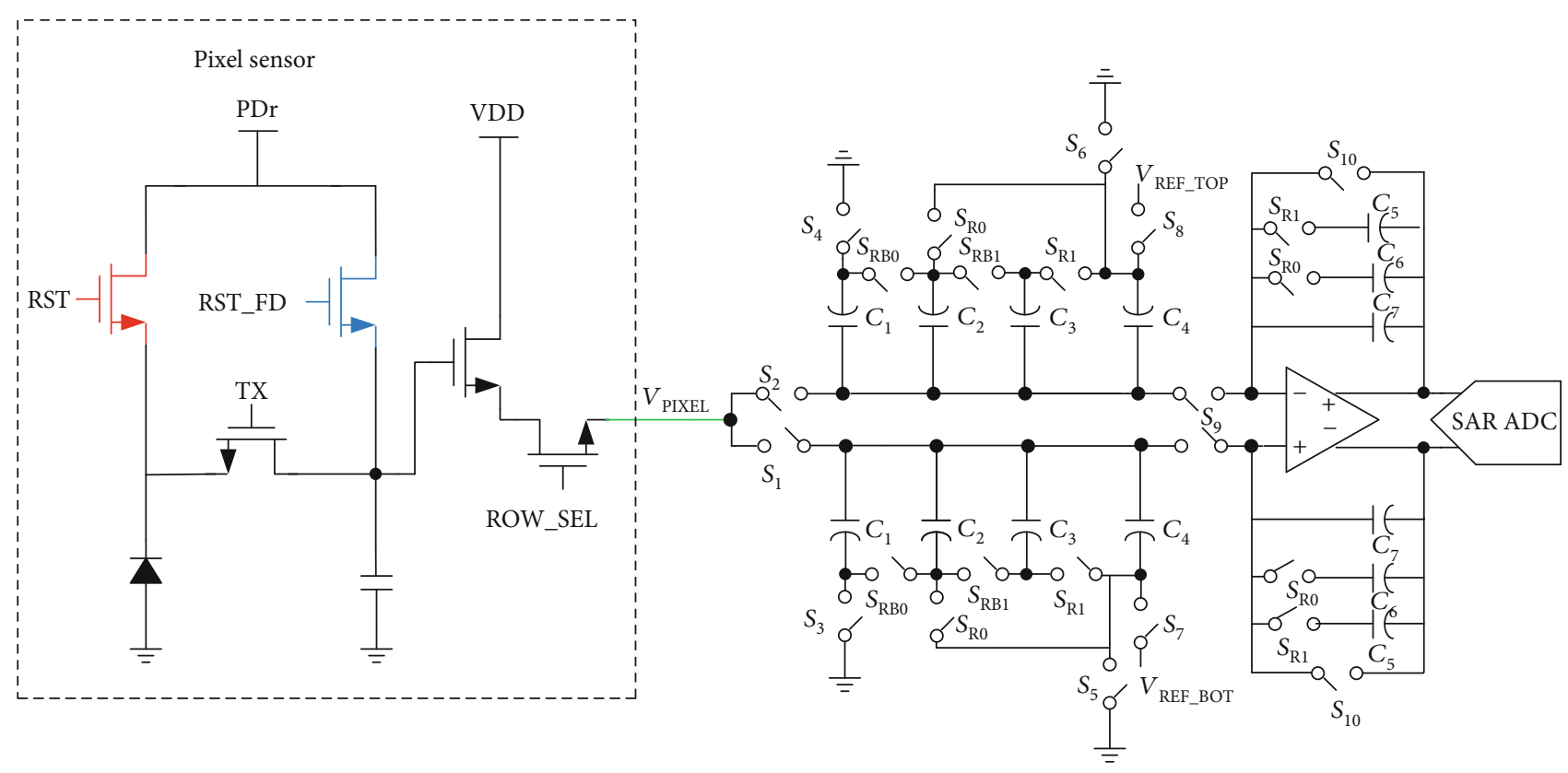

FIGURE 3: The proposed architecture of PGA samples the signal from the 5-T pixel sensor.

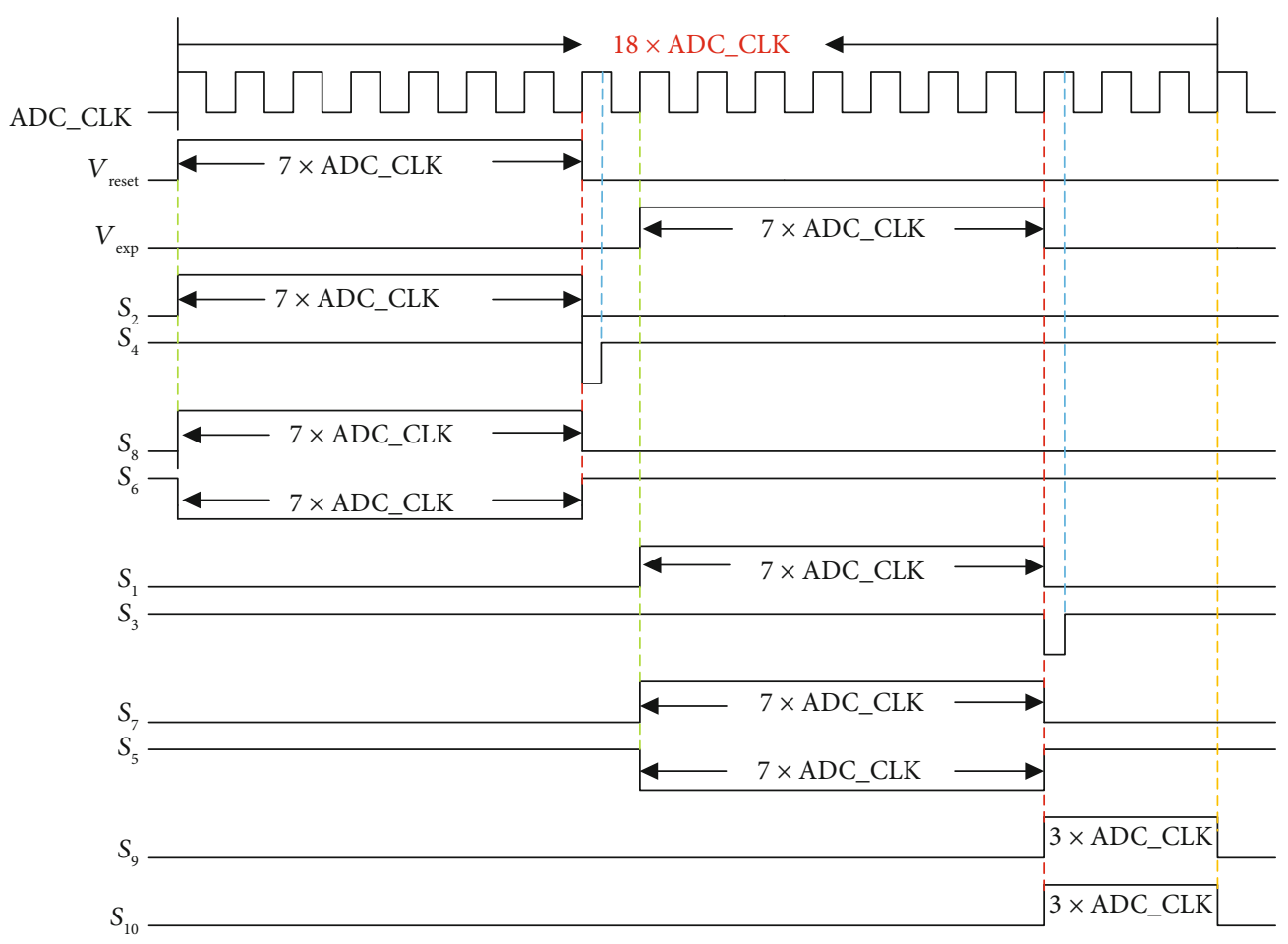

Figure 4: Timing diagram for the PGA.

improve the performance of SAR ADC. What is more, PGA provides the gain of $3 \mathrm{x}, 4 \mathrm{x}$, and $6 \mathrm{x}$, based on the input signal.

The initial shorter conference paper introduces the working principle and results of the CIS chip briefly [7]. Based on the initial paper, this paper is organized as follows, which shows more details of the CIS chip. Section 2 describes the architecture of the system. The operation principle of different modules is discussed in Section 3, including implementa- tion details of ADC and PGA. The simulated and experimental results of the prototype are illustrated in Section 4 . Section 5 concludes this paper.

\section{System Architecture}

Figure 1 shows the architecture of the proposed CIS, which includes pixel array, PGA, ADC, register, row scanner, 
reference generator, reference calibration, Low-Voltage Differential Signaling (LVDS) drivers, and auxiliary circuits. Considering more than one million pixels and the frame rate (50 fps), a column readout circuit is used when a row scanner is applied on selecting a specific row. When the row is determined by a row scanner, a column readout circuit begins to work. The small signal is amplified by PGA and then converted into a digital signal by ADC, which is shifted into register. Finally, the LVDS driver takes on the role of output signal. The proposed CIS works at the speed of $150 \mathrm{KS} / \mathrm{s}$, and the reference clock signal is $2.7 \mathrm{MHz}$.

During the whole process, the function of PGA is to sample exposure signal, reset signal, and provide three gains: $3 \mathrm{x}$, $4 \mathrm{x}$, and $6 \mathrm{x}$. Owing to exposure signal lower than reset signal, which means that the calculated signal for the difference between exposure signal and reset signal is single-ended, the fixed deviation is added to the system for converting a single-ended signal into differential forms, which is consistent with SAR ADC input. What is more, in order to speed the upsampling process, three-way PGA corresponding to three columns of pixel array is adopted instead of sampling signal one by one. The gain of PGA is decided by the size of signal, which should be distinguished before transferred into SAR ADC.

14-bit fully differential SAR ADC is necessary for the proposed CIS, which directly affects the image. The highprecision reference voltage is the guarantee of SAR ADC. As a result, a scaled reference generator is used with a reference calibration module.

The system timing of the proposed CIS is shown in Figure 2. The working cycle of the chip is 18 ADC_CLKs (reference clock signal). During the first 3 ADC_CLKs, a specific row is chosen when SAR ADC samples the signal amplified by PGA. The remained 15 ADC_CLKs mainly include the following work: the conversion of SAR ADC, the signal sampling of PGA, and the data reading. During the sampling process of PGA, the reset signal is sampled first and the exposure signal follows.

\section{Operation Principle and Circuit Implementation}

In this section, the operation principle and circuit implementation of CIS are introduced, which consist of PGA, SAR $\mathrm{ADC}$, and reference voltage self-correction algorithm. Referring to SAR ADC, principle and timing are described. What is more, the core parts of SAR ADC are presented in detail, including comparator and DAC.

3.1. PGA. In the CMOS image sensor system, a pixel sensor which demonstrates the brightness of the outside light depends on the difference between reset signal and exposure signal. The form of reset and exposure signal is voltage, which needed to be sampled and amplified into a suitable size. In order to increase the speed of the readout circuit, reset and exposure signals are sampled by different capacitors and amplified by a differential amplifier.

Based on the principle of pixel array, the reset signal is always higher than the exposure signal, which results in the
TABle 1: The value of capacitors from $C_{1}$ to $C_{7}$.

\begin{tabular}{lrrrrrr}
\hline$C_{1}$ & $C_{2}$ & $C_{3}$ & $C_{4}$ & $C_{5}$ & $C_{6}$ & $C_{7}$ \\
\hline $33 C_{u}$ & $4 C_{u}$ & $4 C_{u}$ & $7 C_{u}$ & $4 C_{u}$ & $4 C_{u}$ & $8 C_{u}$ \\
\hline
\end{tabular}

unipolar difference all the time. The proposed architecture of PGA shown in Figure 3 aims at solving the problem by introducing a fixed deviation. $V_{\text {PIXEL }}$ represents the reset and exposure signals, which is the output signal of the 5-T pixel sensor. With the help of a combination of capacitors $C_{1}, C_{2}, C_{3}$, and $C_{4}$, the unipolar difference between them is converted into bipolar form and averaged over the positive and negative half axes. The single-ended signal is transferred to a differential signal, which is more convenient for SAR ADC. The control action of switches $S_{\mathrm{RB}}$ and $S_{\mathrm{R}}$ is the opposite, which decides the gain of PGA. The switching of $S_{\mathrm{R} 1}$ and $S_{\mathrm{R} 0}$ changes the magnitude of the gain from $3 \mathrm{x}$ to $4 \mathrm{x}$ or $6 \mathrm{x}$. What is more, $V_{\text {REF_TOP }}$ and $V_{\text {REF_BOT }}$ are the reference voltage of SAR ADC, which is applied on PGA as well. The purpose of adopting different reference voltages is to generate the needed deviation.

The working cycle of PGA is 18 ADC_CLKs, which is the same as the system and ADC working cycle. The following analysis of operation principle of PGA is based on the charge. The change on charge represents the switch of working status.

As shown in Figure 4, reset signal which is called $V_{\text {reset }}$ is got by PGA. During the first 7 ADCs, switches $S_{2}, S_{4}$, and $S_{8}$ are on while $S_{6}$ is off on the upper capacitor array part. What is more, switches $S_{\mathrm{RB} 0}$ and $S_{\mathrm{R} 1}$ are on when the lower plates of capacitors $C_{1}$ and $C_{2}$ are connected to the ground and the lower plates of capacitors $C_{3}$ and $C_{4}$ are connected to the $V_{\text {REF_TOP }}$. The total charge $Q_{\mathrm{UP}}$ stored in capacitors $C_{1}, C_{2}$, $C_{3}$, and $C_{4}$ on the upper part is calculated as follows:

$$
Q_{\mathrm{UP}}=\left(C_{1}+C_{2}\right) \cdot\left(V_{\text {reset }}-V_{\mathrm{GND}}\right)+\left(C_{3}+C_{4}\right) \cdot\left(V_{\text {reset }}-V_{\mathrm{REF}_{\mathrm{TOP}}}\right) .
$$

At the $8^{\text {th }}$ ADC_CLK, all switches on the upper part begin to change. The switches $S_{2}$ and $S_{8}$ turn off while the switch $S_{6}$ turns on. What is more, $S_{4}$ turns off first and turns on again in the half of ADC_CLK. The lower plates of $C_{3}$ and $C_{4}$ are connected from $V_{\text {REF_TOP }}$ to the ground. However, the capacitors are not connected to new power and the total charge keeps on. Considering the switch $S_{2}$ is open, the upper plate voltages of capacitors are not regular. Based on the charge conservation and the ignoration of offset, the voltage of the upper plate can be achieved by

$$
\begin{gathered}
Q_{\mathrm{UP}}=\left(C_{1}+C_{2}+C_{3}+C_{4}\right)\left(V_{\mathrm{UP}}-V_{\mathrm{GND}}\right), \\
V_{\mathrm{UP}}=V_{\text {reset }}-\frac{C_{3}+C_{4}}{C_{1}+C_{2}+C_{3}+C_{4}}\left(V_{\mathrm{REF}_{-} \mathrm{TOP}}-V_{\mathrm{GND}}\right) .
\end{gathered}
$$

The latter 7 ADC_CLKs are from 9 to 15 ADC_CLKs. In this period, the exposure signal is sampled by the lower part 


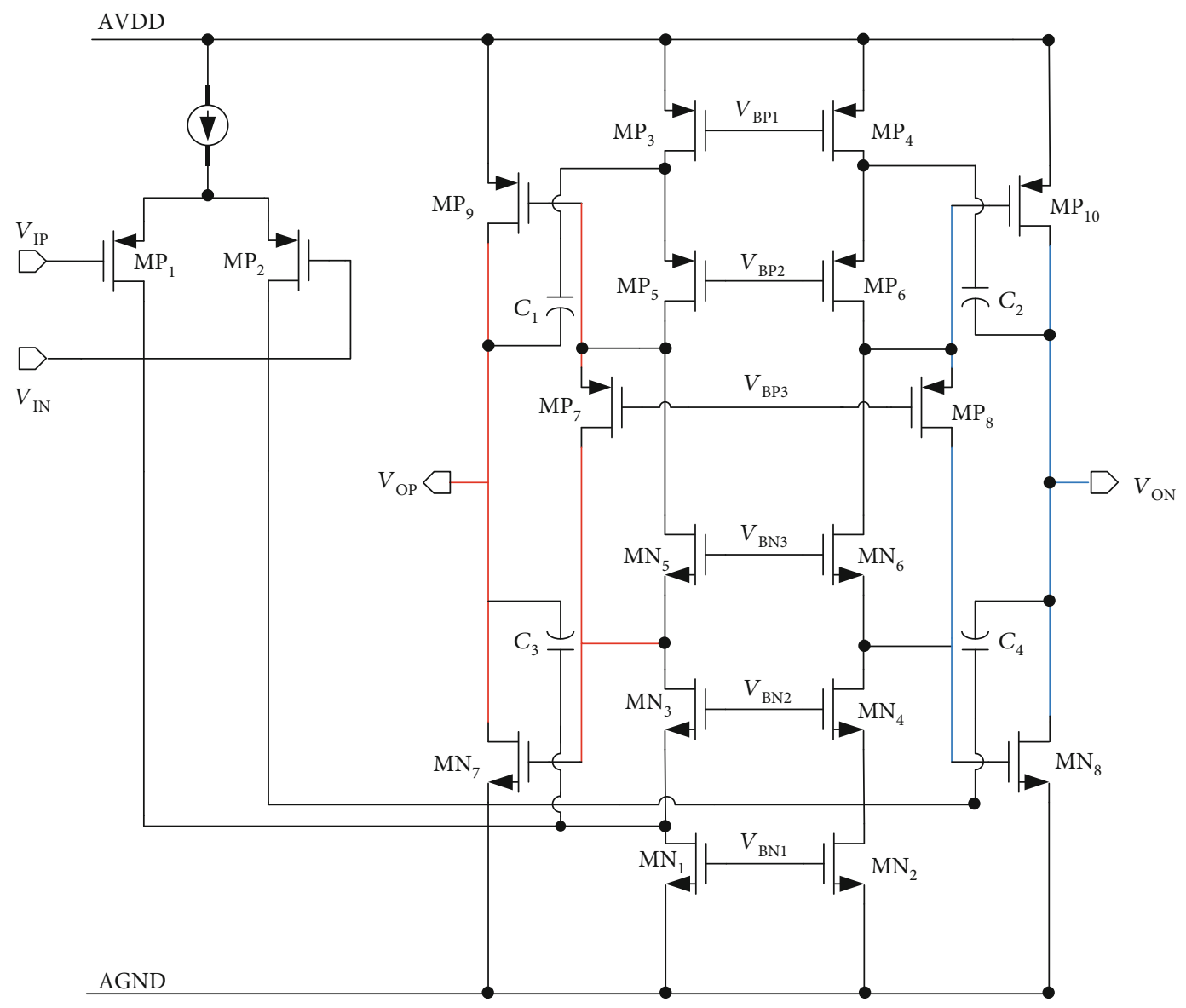

Figure 5: Architecture of operational amplifier.

of a circuit. The principle and process of sampling are the same as the reset signal. At the beginning, the switches $S_{1}$, $S_{3}$, and $S_{7}$ are closed and $S_{5}$ is open. The charge $Q_{\mathrm{DN}}$ sampled in this procedure is

$$
Q_{\mathrm{DN}}=\left(C_{1}+C_{2}\right) \cdot\left(V_{\exp }-V_{\mathrm{GND}}\right)+\left(C_{3}+C_{4}\right) \cdot\left(V_{\text {exp }}-V_{\mathrm{REF} \_\mathrm{TOP}}\right) .
$$

And then, the switch $S_{5}$ turns on and $S_{1}, S_{3}$, and $S_{7}$ turn off. Finally, $S_{1}$ and $S_{7}$ keep off and $S_{3}$ and $S_{5}$ keep on, where the state of $S_{3}$ is from open to closed. Through this process, the following equations can be obtained:

$$
\begin{gathered}
Q_{\mathrm{DN}}=\left(C_{1}+C_{2}+C_{3}+C_{4}\right)\left(V_{\mathrm{DN}}-V_{\mathrm{GND}}\right), \\
V_{\mathrm{DN}}=V_{\text {reset }}-\frac{C_{3}+C_{4}}{C_{1}+C_{2}+C_{3}+C_{4}}\left(V_{\mathrm{REF}_{-} \mathrm{BOT}}-V_{\mathrm{GND}}\right) .
\end{gathered}
$$

Combining equations (1)-(6), the differential signal sent into the amplifier is described by the following equations:

$$
V_{\mathrm{IN}}=V_{\mathrm{DN}}-V_{\mathrm{UP}},
$$

$$
V_{\mathrm{IN}}=\left(V_{\text {exp }}-V_{\text {reset }}\right)+\frac{C_{3}+C_{4}}{C_{1}+C_{2}+C_{3}+C_{4}}\left(V_{\mathrm{REF}_{-} \mathrm{TOP}}-V_{\mathrm{GND}}\right) .
$$

According to equation (8), apart from the difference between $V_{\text {exp }}$ and $V_{\text {reset }}$, the fixed deviation is introduced as expected, which realizes the goal of making the signal spread uniformly. After sampling is finished, switches $S_{9}$ and $S_{10}$ turn on, which lasts 3 ADC_CLK.

During the phase, the function of $S_{\mathrm{R} 0}$ and $S_{\mathrm{R} 1}$ is to choose different gains which is called amplify phase as well. The value of capacitors used in the PGA is shown in Table 1. The calculation formula of gain is

$$
V_{\mathrm{IN}}=\frac{C_{1}+C_{2}+C_{3}+C_{4}}{C_{5}+C_{6}+C_{7}}
$$

If switches $S_{\mathrm{R} 0}$ and $S_{\mathrm{R} 1}$ both turn on, the gain of PGA is $6 \mathrm{x}$. While if one of them turns off, the gain of PGA is $4 \mathrm{x}$. Once both are closed, the smallest gain $3 \mathrm{x}$ is achieved under this situation.

As a significant part of PGA, the operational amplifier needs to be paid enough attention to. Figure 5 shows the architecture of amplifier, which needs high gain, enough bandwidth, strong driving capability, and low noise. The 


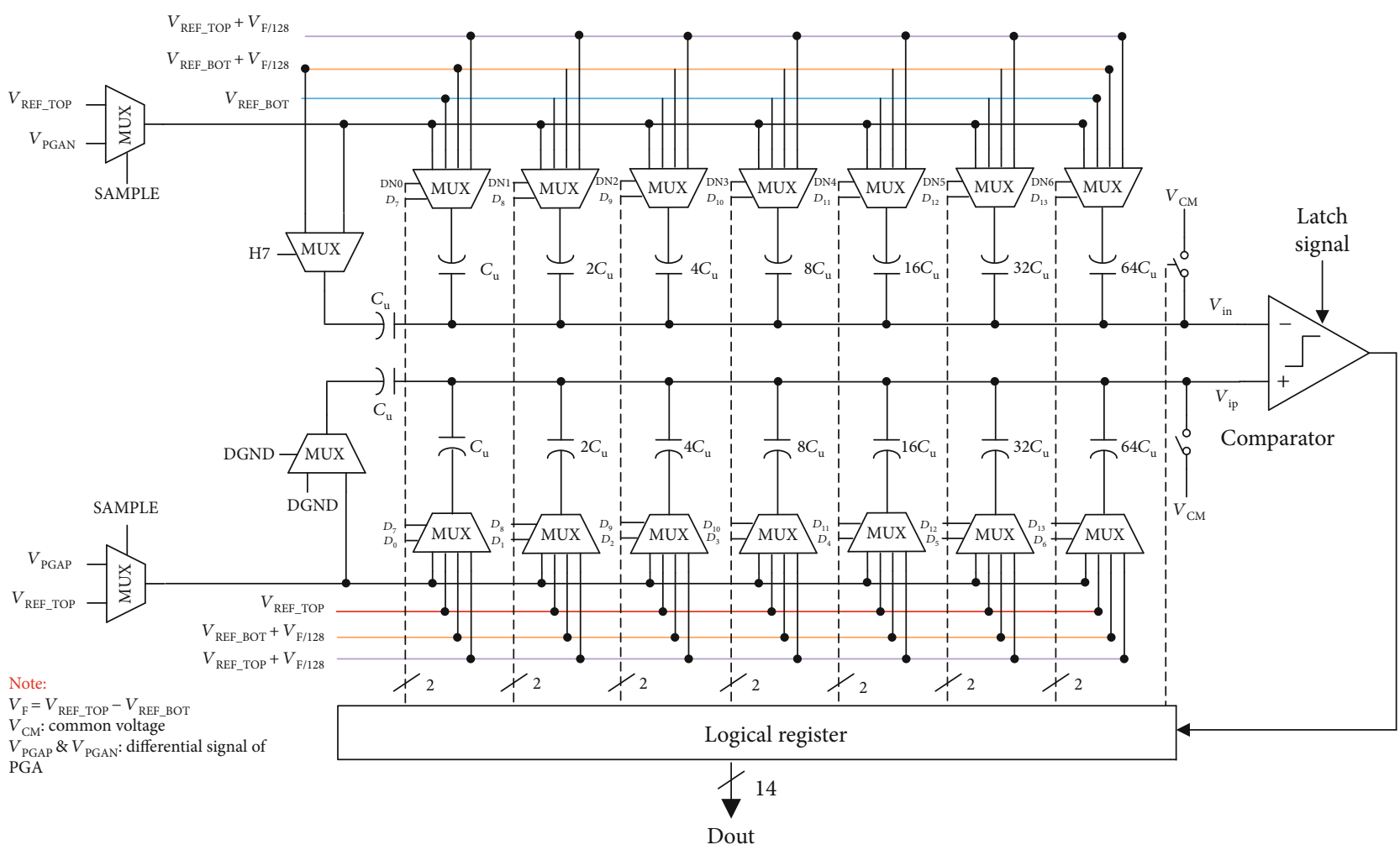

FIgURE 6: The architecture of the proposed SAR ADC.

proposed amplifier contains two stages: amplifying stage and driving stage, aimed at providing enough gain and increasing the ability of driving. Compared with other architectures of amplifier, the wider input common mode range and larger output swing are the advantages of folding cascode operational amplifier, which is applied in this situation and provides major gain. The cascode stage makes the $R_{\text {out }}$ increase, which leads to high gain.

The driving stage is the floating-biased class $\mathrm{AB}$ output stage, having a strong driving ability. The size of followingup capacitor arrays used in the SAR ADC is large, which puts forward higher requirements for the driver of the input stage. Considering the high power consumption of folding cascode amplifier, the floating biased transistors $\mathrm{MN}_{5}, \mathrm{MN}_{6}, \mathrm{MP}_{7}$, and $\mathrm{MP}_{8}$ are utilized to decrease and stabilize quiescent current of the output stage as analyzed in [8]. The overall gain of the two-stage amplifier is

$$
\begin{aligned}
A_{\mathrm{op}}= & g_{\mathrm{MP} 1} \bullet\left(\left(g_{\mathrm{MN} 3} \bullet r_{o, \mathrm{MN} 3} \bullet\left(r_{o, \mathrm{MN} 1} \| r_{o, \mathrm{MP} 1}\right)\right) \|\left(g_{\mathrm{MP} 5} \bullet r_{o, \mathrm{MP} 3} \bullet r_{o, \mathrm{MP} 5}\right)\right) \\
& \cdot\left(g_{\mathrm{MN} 7}+g_{\mathrm{MP} 9}\right) \bullet\left(r_{o, \mathrm{MN} 7} \| r_{o, \mathrm{MP} 9}\right) .
\end{aligned}
$$

When it comes to noise, the high noise is always the drawback of folding cascode amplifier, as depicted in [9]. However, the operational amplifier of this structure meets design requirements. In order to improve the performance of noise, PMOS is adopted as input, which is better than NMOS on noise. What is more, larger value of $g_{\mathrm{MP} 1}$ and smaller value of $g_{\mathrm{MN} 1}$ and $g_{\mathrm{MP} 3}$ are chosen to reduce the noise. Under the circumstance, the value of W/L needs to be weighed carefully, which is important for improving the SNR of SAR ADC

3.2. SAR ADC. The most important module of CIS is SAR ADC, which is placed after PGA. The function of SAR $\mathrm{ADC}$ is to convert the analog signal amplified by PGA into the digital signal.

The sampling speed of SAR ADC required by the system is $150 \mathrm{KS} / \mathrm{s}$, and the resolution of SAR ADC is 14 bits. Normally, the differential input method is selected to suppress the interference of common mode factors and the charge redistribution theory is applied on SAR ADC, which was first proposed by McCreary and Gray [10]. Combing the above two points, the architecture of double reference voltage SAR ADC is widely used. If the resolution of SAR ADC is beyond 10 bits, the capacitor array will be very large, taking up unexpected area in the situation. In order to avoid a huge capacitor array, the two-step scaled-reference SAR ADC is put forward, which is based on the charge redistribution theory as well. This structure was first proposed by South Korea's Shin for CMOS image sensor applications [11]. Twostep scaled reference contains four reference voltages, and the increased reference voltage is to reduce the area of the capacitor array.

3.2.1. Principle and Timing. The architecture of the proposed SAR ADC is shown in Figure 6, containing DAC, comparator, and SAR Logic. Compared with double reference voltages, two-step scaled reference keeps on the size of 


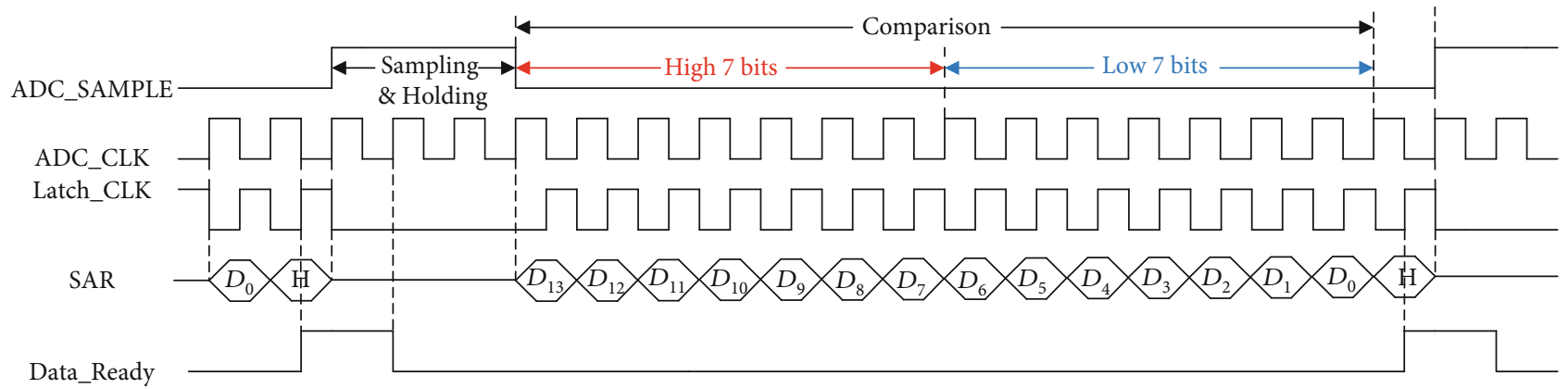

FIgURE 7: Timing diagram of SAR ADC.

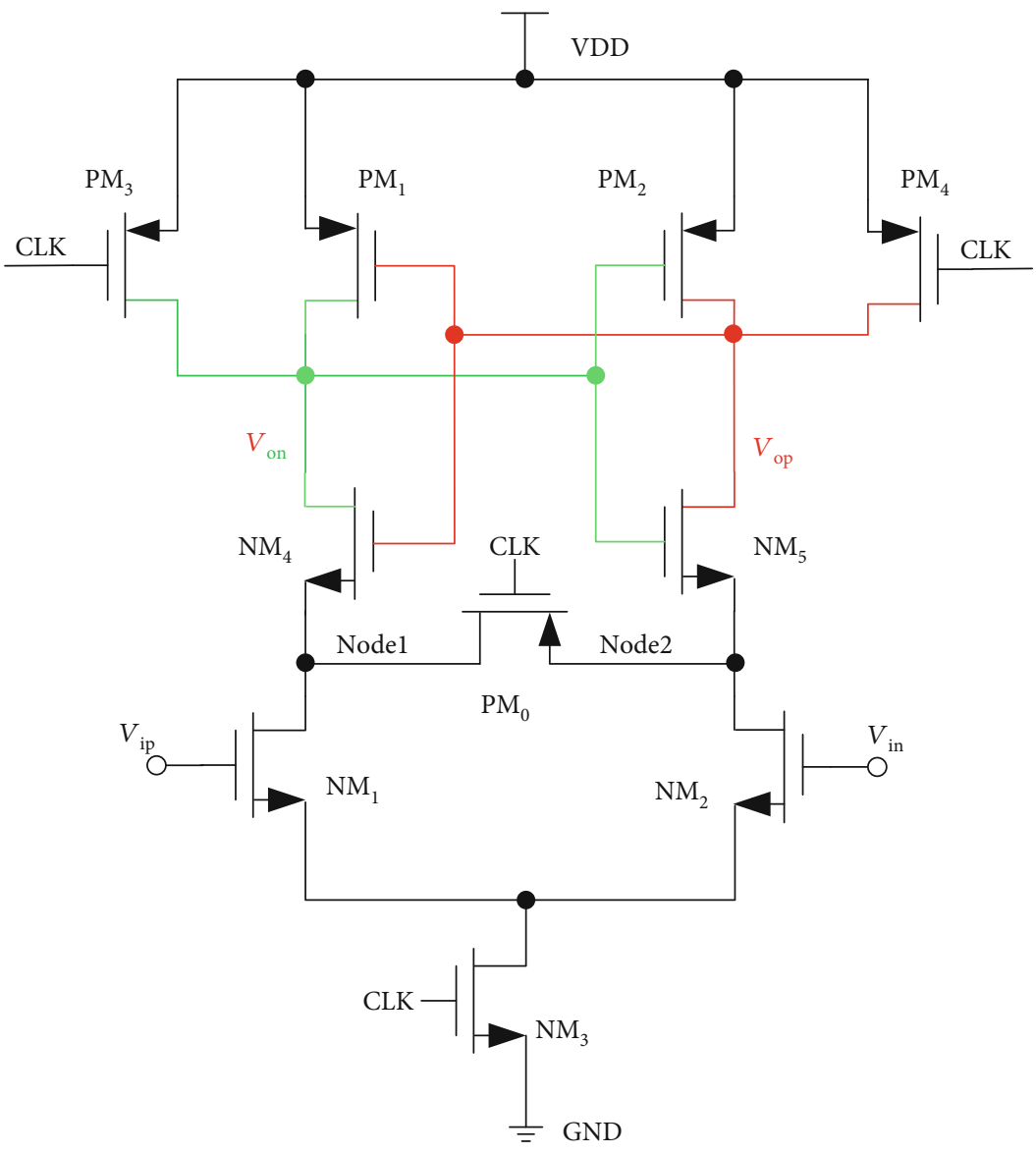

FIGURE 8: StrongARM latch topology.

capacitor array with the complex conversion process. Use the same size capacitor array, but the resolution has changed from the previous 7 bits to 14 bits, which is the core advantage of the architecture. On the other hand, the requirement of precision of reference voltage is increasing. The added reference voltages $V_{\text {REF_TOP }}+V_{F} / 128$ and $V_{\text {REF_BOT }}+V_{F} / 128$ need higher accuracy compared with $\bar{V}_{\text {REF_TOP }}$ and $V_{\text {REF_BOT }} \cdot V_{\text {REF_TOP }}$ and $V_{\text {REF_BOT }}$ are the reference voltages used in the first stage while the $V_{\text {REF_TOP }}+V_{F} / 128$ and $V_{\text {REF_BOT }}+V_{F} / 128$ are applied on the second stage. The value of $V_{F}$ is

$$
V_{F}=V_{\text {RFF_TOP }}-V_{\text {RFF_BOT }} .
$$

$V_{\mathrm{PGAP}}$ and $V_{\mathrm{PGAN}}$ are the output signals of PGA, which are the same as $V_{\mathrm{OP}}$ and $V_{\mathrm{ON}}$ shown in Figure 5. $V_{\mathrm{CM}}$ is the common voltage of the input signal of SAR ADC.

The whole process includes four parts: sampling, holding, comparison, and output. The architecture adopts lower plates of capacitors to sample. During the sampling phase, 


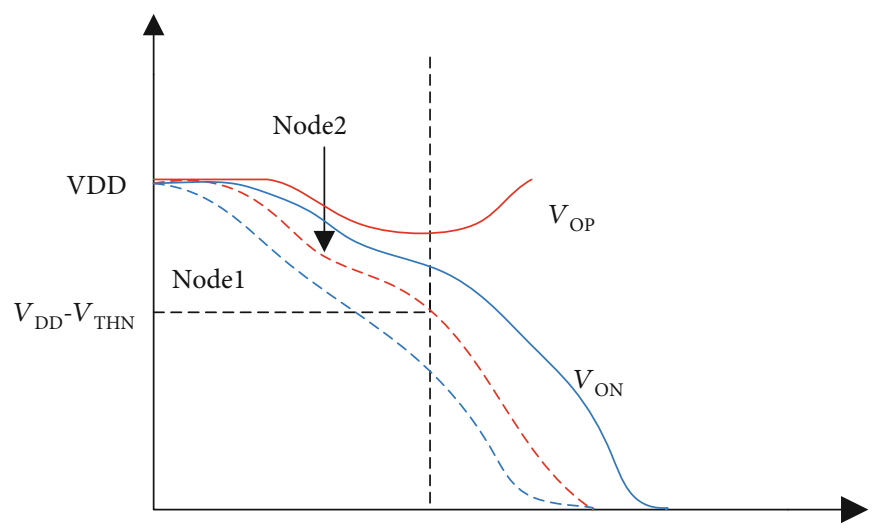

FIgURE 9: The voltage change of the whole process.

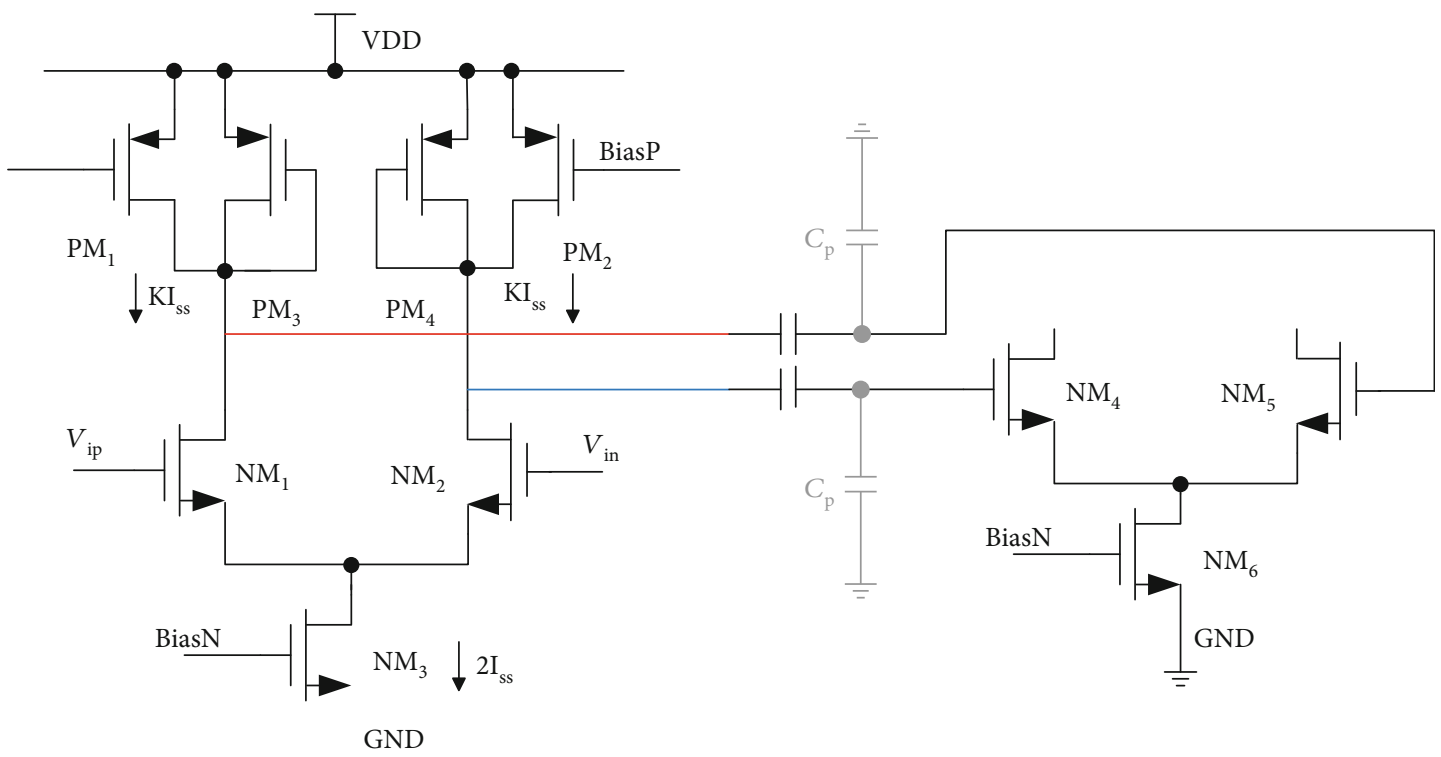

FIgURE 10: The architecture of preamplifier.

$V_{\text {PGAP }}$ and $V_{\text {PGAN }}$ are chosen by MUX when all upper plates of capacitors are connected to $V_{\mathrm{CM}}$. The signal $V_{\mathrm{PGAP}}$ and $V_{\text {PGAN }}$ are sampled by the capacitor array because all lower plates of capacitors are connected to $V_{\text {PGAP }}$ and $V_{\text {PGAN }}$. The charge $Q_{s}$ stored in all capacitors is calculated as

$$
Q_{s}=-\left(V_{\mathrm{PGAP}}-V_{\mathrm{PGAN}}\right) * 2^{7} C_{u}
$$

Converting the input voltage into the form of charge is the principle of sampling. In order to hold the sampled charge, the upper plates of capacitors do not connect to the $V_{\mathrm{CM}}$. The lower plates of capacitors in the negative part are connected to the $V_{\text {REF_TOP }}$ when the lower plates of capacitors in the positive part are connected to the $V_{\text {REF_BOT }}$. After finishing the procedure, according to the charge conservation theory, the following equations are obtained:

$$
\begin{aligned}
& Q_{\mathrm{HP}}=\left(V_{\mathrm{ip}}-V_{\mathrm{REF}_{\mathrm{BOT}}}\right) * 2^{7} C_{u}, \\
& Q_{\mathrm{HN}}=\left(V_{\mathrm{in}}-V_{\mathrm{REF}_{\mathrm{TOP}}}\right) * 2^{7} C_{u},
\end{aligned}
$$

$$
Q_{H}=Q_{\mathrm{HP}}-Q_{\mathrm{HN}} \cdot
$$

Combining equations (12)-(15), the input signal of comparator is achieved:

$$
\begin{gathered}
V_{\text {ip }}=V_{\text {REF_BOT }}-V_{\text {PGAP }}, \\
V_{\text {in }}=V_{\text {REF_TOP }}-V_{\text {PGAN }}, \\
V_{\text {IN }}=V_{\text {ip }}-V_{\text {in }}=V_{\text {REF_BOT }}-V_{\text {PGAP }}-\left(V_{\text {REF_TOP }}-V_{\text {PGAN }}\right) .
\end{gathered}
$$

Once the holding phase is finished, SAR ADC enters the comparison phase, which contains two parts: high 7-bit conversion and low 7-bit conversion. The reference voltages of high 7-bit conversion are $V_{\text {REF_TOP }}$ and $V_{\text {REF_BOT }}$ while $V_{\mathrm{REF} \_\mathrm{TOP}}+V_{F} / 128$ and $V_{\mathrm{REF} \_ \text {BOT }}+V_{F} / 128$ are the reference voltages of low 7-bit conversion. Controlling the connection of lower plates of capacitor array to adjust the input signal of comparator and then get the corresponding code according 


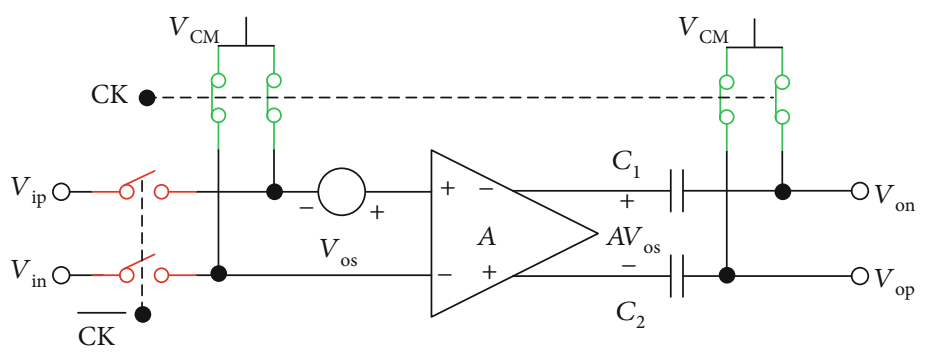

(a) Reset phase

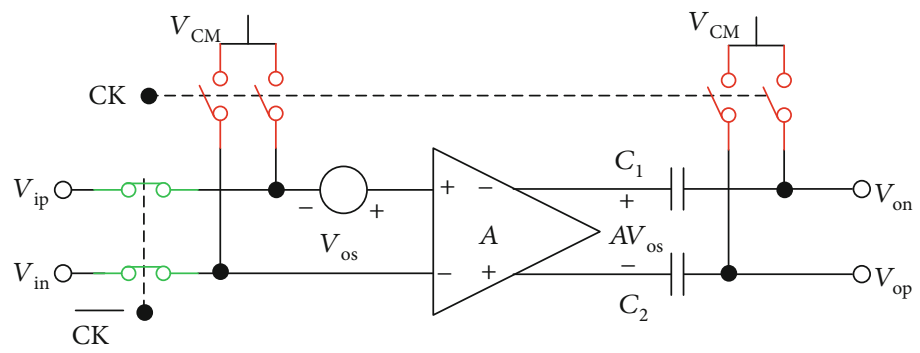

(b) Amplification phase

FIgURE 11: Offset cancellation circuit.

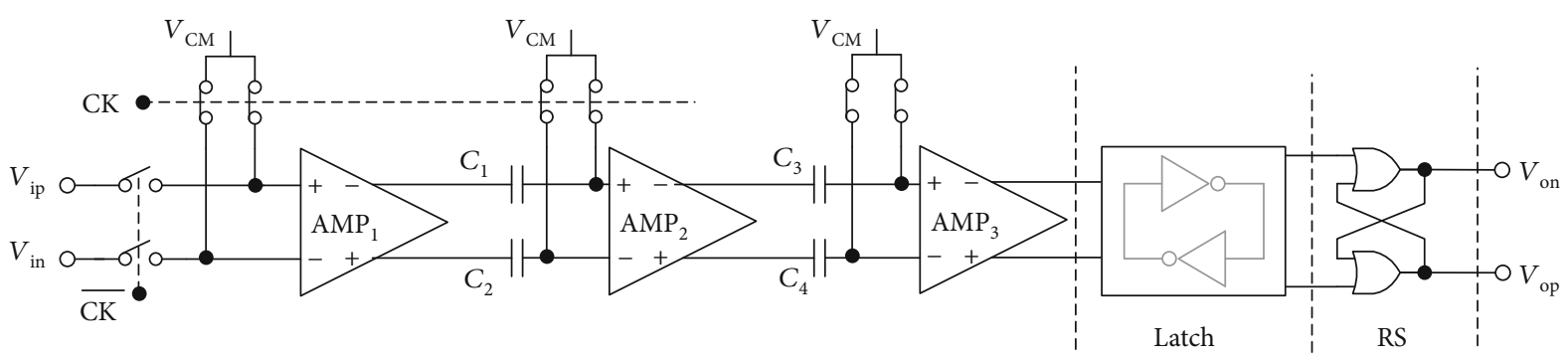

FIGURE 12: The complete structure of comparator.

to the comparison result is the core working principle of comparison.

During the high 7-bit conversion, the $\operatorname{MSB}\left(D_{13}\right)$ is taken for example and the others are the same working process. At the beginning, the lower plate of highest capacitance $\left(64 C_{u}\right)$ in the positive side is connected from $V_{\text {REF_BOT }}$ to $V_{\text {REF_TOP }}$ and the lower plate of the highest capacitor $\left(64 C_{u}\right)$ in the negative side is connected to $V_{\text {REF_BOT }}$. The other plates of capacitance keep on the current states. The change of charge $Q_{c}$ stored in the capacitor array is calculated as follows:

$$
\begin{aligned}
Q_{\mathrm{c}}= & \left(V_{\mathrm{REF}_{\mathrm{TOP}}}-V_{\mathrm{REF}_{\mathrm{BOT}}}\right) * 2^{6} C_{u}-\left(V_{\mathrm{REF}_{\mathrm{BOT}}}-V_{\mathrm{REF}_{\mathrm{TOP}}}\right) \\
& * 2^{6} C_{u}=2 *\left(V_{\mathrm{REF}_{\mathrm{TOP}}}-V_{\mathrm{REF}_{\mathrm{BOT}}}\right) * 2^{6} C_{u} \\
= & \left(V_{\mathrm{REF}_{\mathrm{TOP}}}-V_{\mathrm{REF}_{\mathrm{BOT}}}\right) * 2^{7} C_{u} .
\end{aligned}
$$

The input signal of comparator $V_{\mathrm{IN}}$ follows with $Q_{\mathrm{c}}$ :

$$
V_{\mathrm{IN}}=-\left(V_{\mathrm{PGAP}}-V_{\mathrm{PGAN}}\right)
$$

If the $V_{\mathrm{IN}}$ is negative, the result of comparator is 0 , which means $V_{\text {PGAP }}$ is larger than $V_{\text {PGAN }}$ and the SAR Logic should control the switch to keep on. Otherwise, the result of comparator is 1 . The lower plate of highest capacitance $\left(64 C_{u}\right)$ in the positive side is connected to $V_{\mathrm{REF}}$ вот instead of $V_{\text {REF_TOP }}$, and the negative side is the opposite, which also represents $V_{\text {PGAP }}$ which is smaller than $V_{\text {PGAN }}$. The rest 6 bits work in the same way.

The conversion of low 7 bits is different from high 7 bits. When the conversion of the $7^{\text {th }}$ bit is finished, the lower plate of dummy capacitor in the negative side is connected to $V_{\text {REF_BOT }}+V_{F} / 128$ and the lower plates of other capacitors are connected to $V_{\mathrm{REF} \_ \text {BOT }}+V_{F} / 128$ or $V_{\mathrm{REF}-\mathrm{TOP}}+V_{F} / 128$, which are determined by the original state and increases by $V_{F} / 128$. The purpose of the switch action is to keep the value of $V_{\text {IN }}$ on with the increasement of connected voltages of capacitors in the negative side and decrease of connected voltage of dummy capacitor. The decreased charge $Q_{D E C}$ and $Q_{\mathrm{INC}}$ can be expressed as

$$
Q_{\text {DEC }}=\left(V_{\text {REF_TOP }_{-}}-\left(V_{\mathrm{REF}_{\text {BOT }}}-\frac{V_{F}}{128}\right) \cdot C_{u}=\left(V_{F}-\frac{V_{F}}{128}\right) \cdot C_{u}\right. \text {, }
$$




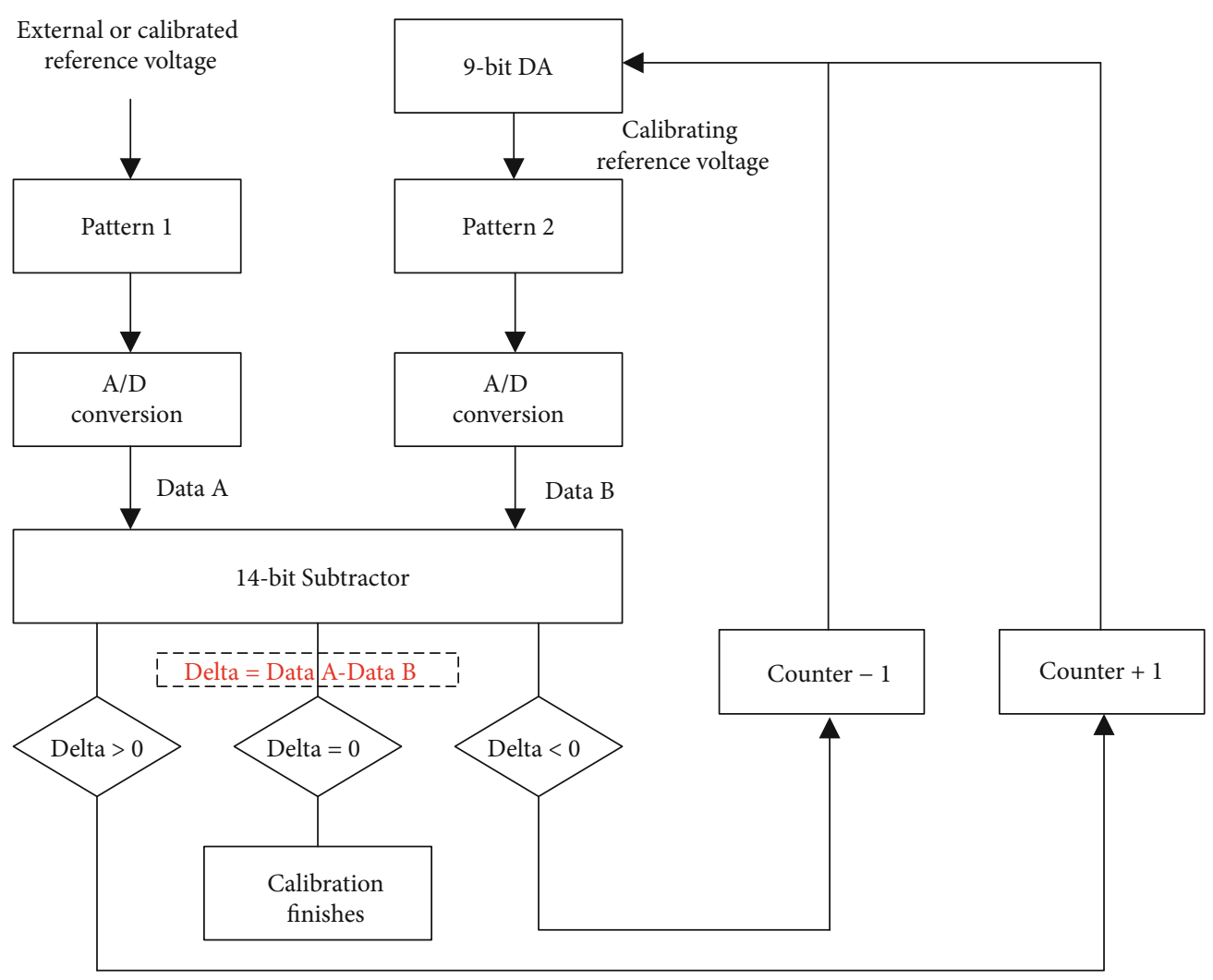

FIGURE 13: Flowchart of the self-calibration algorithm.

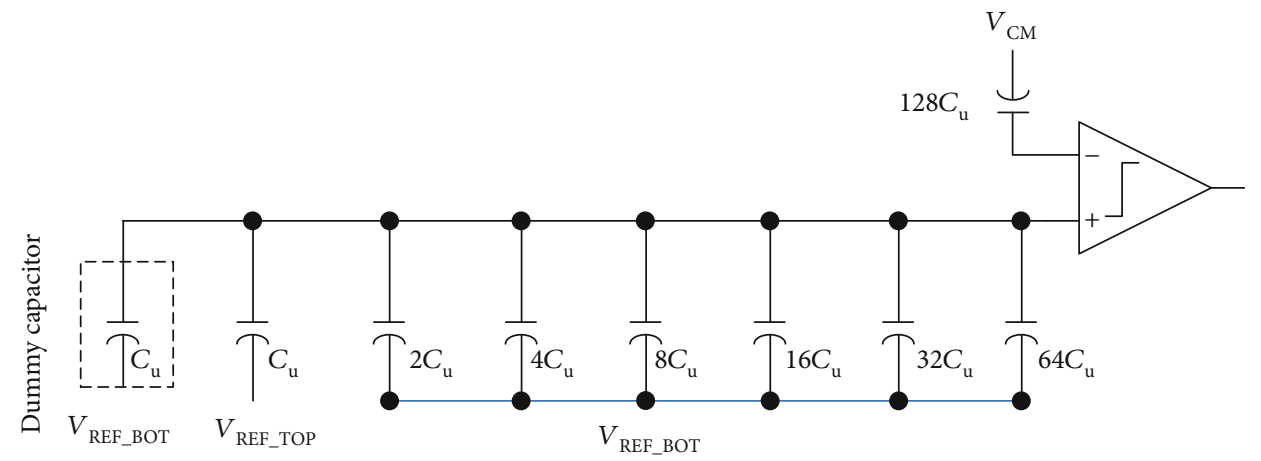

(a)

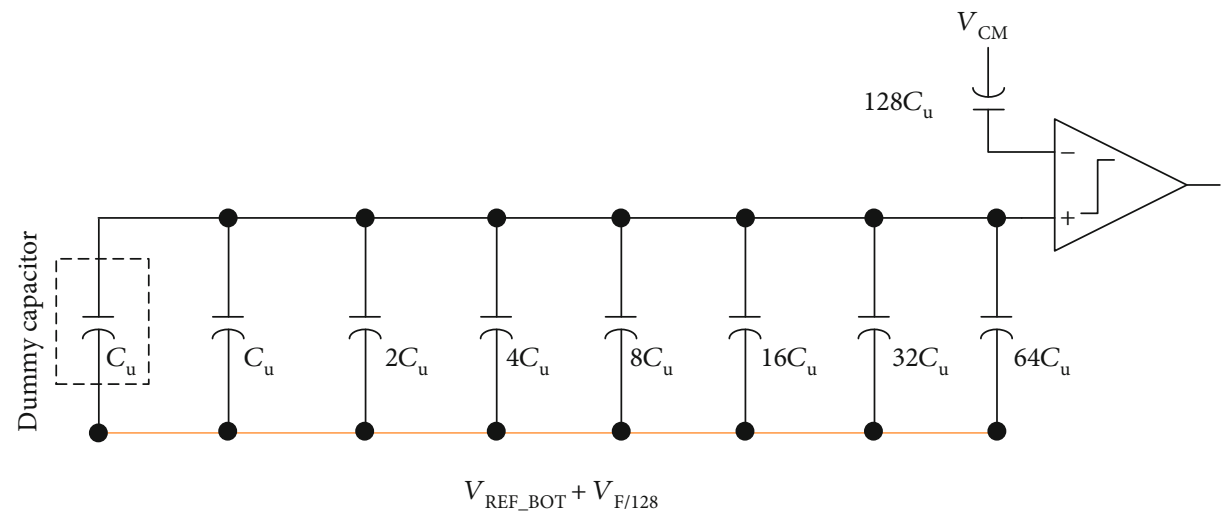

(b)

FIgURe 14: (a) $V_{\text {REF_BOT }}+V_{F} / 128$ calibration pattern 1 and (b) $V_{\text {REF_BOT }}+V_{F} / 128$ calibration pattern 2. 


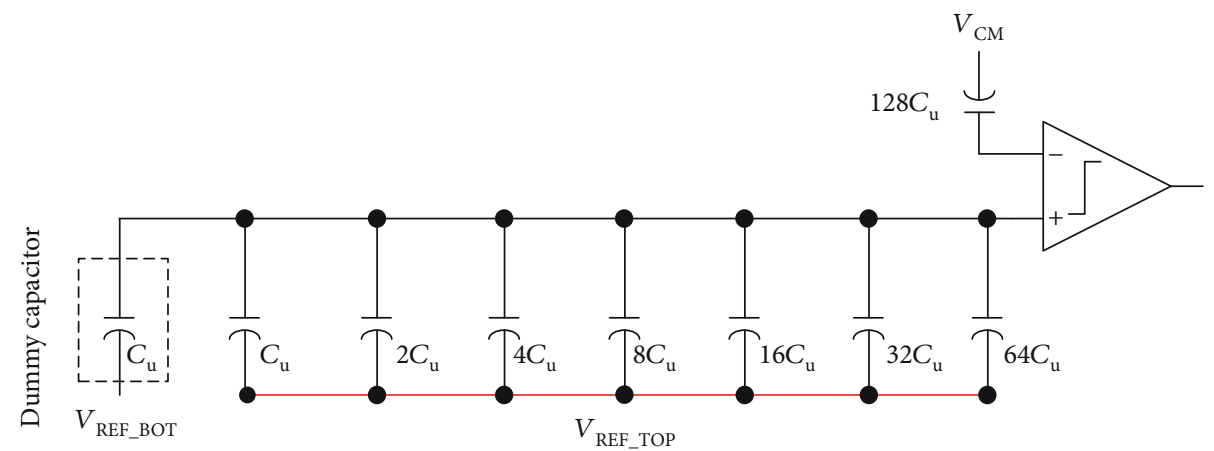

(a)

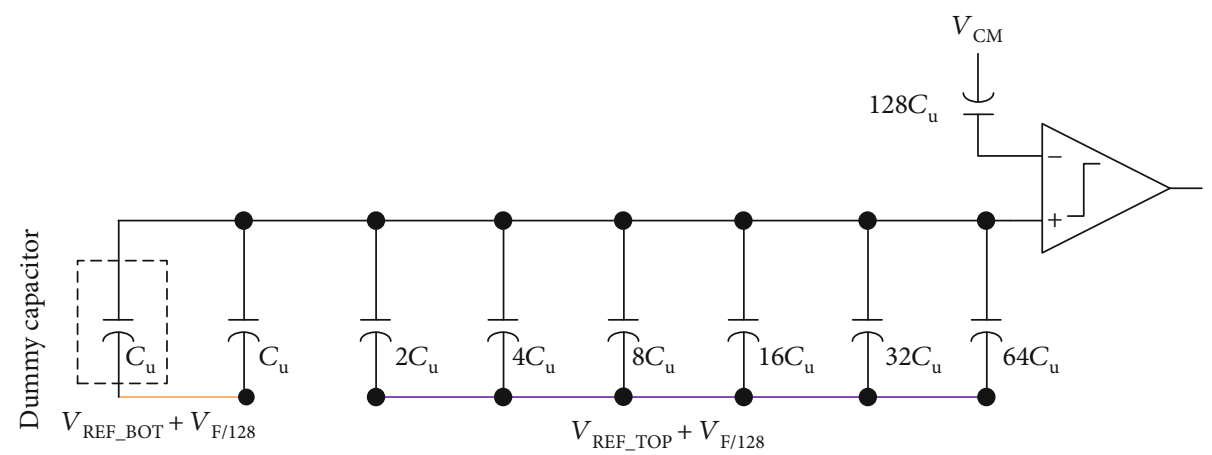

(b)

FIgURE 15: (a) $V_{\text {REF_TOP }}+V_{F} / 128$ calibration pattern 1 and (b) $V_{\text {REF_TOP }}+V_{F} / 128$ calibration pattern 2.

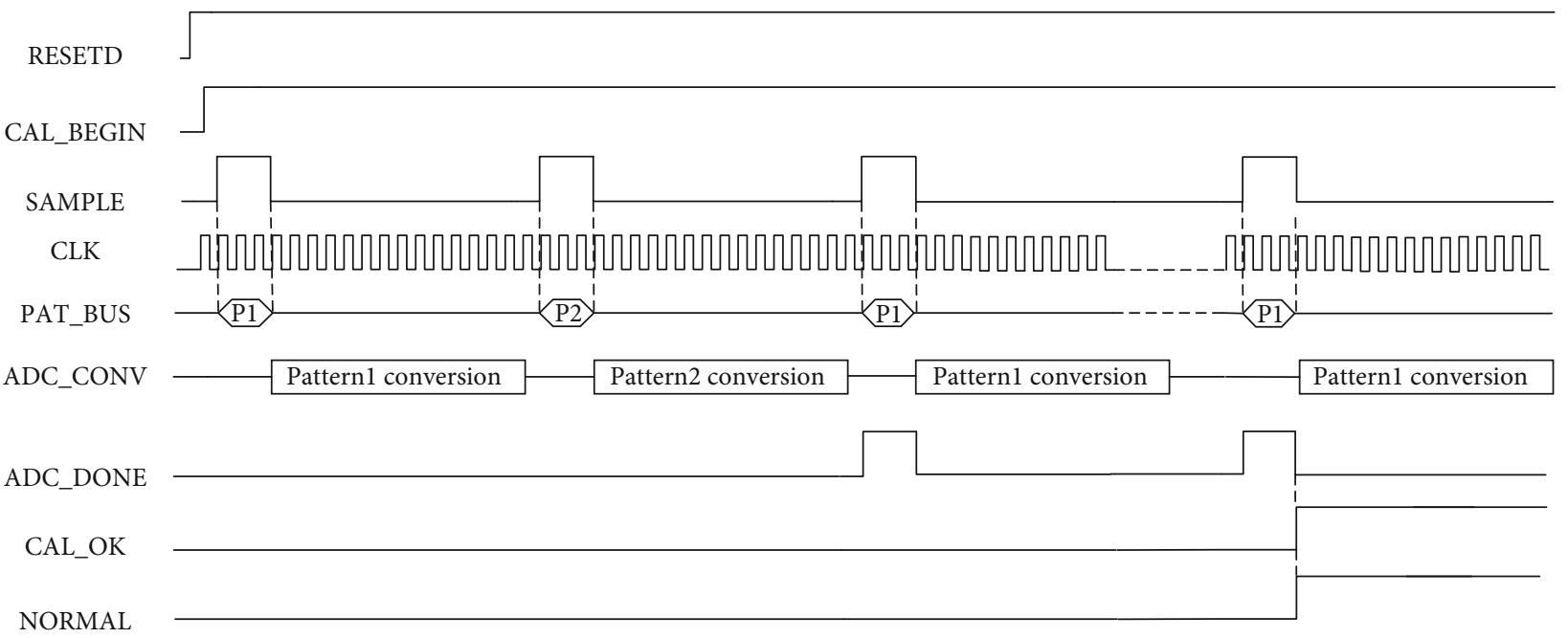

FIgURE 16: The timing block of calibration.

$Q_{\mathrm{INC}}=\frac{V_{F}}{128} \cdot\left(\sum_{n=6}^{0} \frac{1}{2^{n}} \cdot C_{u}\right)=\left(V_{F}-\frac{V_{F}}{128}\right) \cdot C_{u}$.

According to equations (21) and (22), $Q_{\mathrm{DEC}}$ and $Q_{\mathrm{INC}}$ are equal, which means the value of $V_{\text {IN }}$ does not change. At this time, the requirements of low 7-bit conversion are met. In order to explain the process, the comparison of $6^{\text {th }}$ bit is taken for example. Because the low 7-bit conversion and high 7-bit conversion use the same capacitor array, the procedure is similar. What is more, the switch action of lower 7 bits is based on the high 7 bits. For instance, the conversion of $6^{\text {th }}$ bit is related to $13^{\text {th }}$ bit. If $D_{13}$ is 0 , which means the lower plate of the highest capacitance $\left(64 C_{u}\right)$ in the positive side is connected to $V_{\text {REF_BOT }}$ and the lower plate of the highest capacitance $\left(64 C_{u}\right)$ in the negative side is connected to $V_{\text {REF_TOP }}+V_{F} / 128$, the connection voltage of lower plate of the highest capacitance $\left(64 C_{u}\right)$ in the positive side will increase by $V_{F} / 128$ and the connection voltage in the negative side will decrease by $V_{F} / 128$. The increased charge 


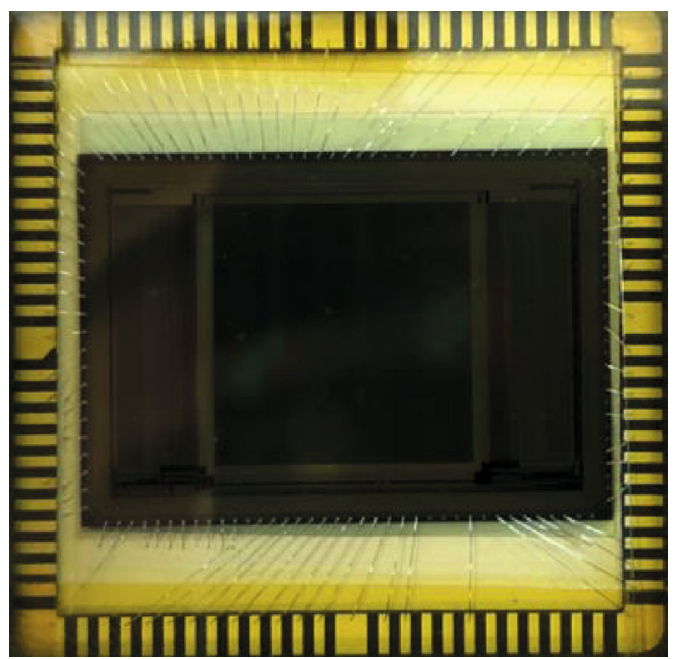

FIGURE 17: The photograph of the chip.

$Q_{\text {INC }}$ and increased voltage of input signal $\Delta V$ are achieved:

$$
\begin{gathered}
Q_{\mathrm{INC}}=\left(\frac{V_{F}}{128}-\left(-\frac{V_{F}}{128}\right)\right) \cdot 64 C_{u}=C_{u} \cdot V_{F}, \\
\Delta V=\frac{V_{F}}{128} .
\end{gathered}
$$

If $D_{13}$ is 1 , which means the lower plate of the highest capacitance $\left(64 C_{u}\right)$ in the positive side is connected to $V_{\text {REF_TOP }}$ and the lower plate of the highest capacitance $\left(64 C_{u}\right)$ in the negative side is connected to $V_{\text {REF_BOT }}+V_{F} /$ 128. The process is similar to the situation of $D_{13}=1$. The change on charge and the input signal are the same as well. The result of comparison determines the switch actions, which has been explained above. The voltage $V_{D 6}$ represented by the capacitor array during the process is calculated:

$$
V_{D 6}=-V_{\mathrm{IN}}+\left(-1+\sum_{n=13}^{6} \frac{1}{2^{D_{n}}}\right) \cdot\left(V_{\mathrm{REF}_{\mathrm{TOP}}}-V_{\mathrm{REF}_{\mathrm{BOT}}}\right) .
$$

The last six bits adopts the same working process. Combining the conversion of high 7 bits and low 7 bits, the analog signal sampled by PGA is converted into 14-bit digital code.

The timing diagram of SAR ADC is shown in Figure 7. The reference clock is still ADC_CLK. The whole procedure occupies 18 ADC_CLKs. The 3 ADC_CLKs are used for sampling while 14 ADC_CLKs are adopted for comparison and the function of the last ADC_CLK is to output the results. During the comparison, the comparator begins to compare at the rising edge of ADC_CLK and latch the signal at the following edge of ADC_CLK. After latching the last comparison, the signal DATA_Ready turns to a high level and keeps on before the first ADC_CLK finishes.

3.2.2. Comparator. The comparator almost decides the speed of SAR ADC. In order to speed up the comparison, the StrongARM Latch topology is used, which is explained in [12]. The
StrongARM latch topology is not only good at speed but also expert in saving power. The circuit is shown in Figure 8.

The StrongARM latch is based on positive feedback, which includes two working stages: reset phase and regeneration phase. At the beginning, the CLK is low. $\mathrm{NM}_{1}$ and $\mathrm{NM}_{2}$ are off while Node1 and Node 2 are connected together to keep the same voltage for resetting. The output $V_{\text {op }}$ and $V_{\text {on }}$ are reset to the power supply voltage by $\mathrm{PM}_{3}$ and $\mathrm{PM}_{4}$, respectively. What is more, $\mathrm{PM}_{1}, \mathrm{PM}_{2}, \mathrm{NM}_{4}$, and $\mathrm{NM}_{5}$ are off. When in the regeneration stage, CLK is high and the current flows through $\mathrm{NM}_{1}$ and $\mathrm{NM}_{2}$. Assuming that $V_{\mathrm{ip}}>V_{\text {in }}$, the current flowing through $\mathrm{NM}_{1}$ is larger than $\mathrm{NM}_{2}$, causing the voltage of Node1 to drop faster than Node2. When the voltages of Node1 and Node2 arrive at $V_{\mathrm{DD}}-V_{\mathrm{THN}}, \mathrm{NM}_{4}$ and $\mathrm{NM}_{5}$ turn on. Because Nodel first reaches $V_{\mathrm{DD}}-V_{\mathrm{THN}}$ , $V_{\text {op }}$ begins to drop, which also leads to the dropping speed of $V_{\text {op }}$ slow down. Positive feedback is formed and $V_{\text {op }}$ becomes $V_{\mathrm{DD}}$ finally. The loop gain $A_{p}$ of the positive feedback loop is

$A_{p}=\left(g_{\mathrm{mn} 4}+g_{\mathrm{mp} 1}\right) \cdot\left(r_{\mathrm{on} 4} \| r_{\mathrm{op} 1}\right) \cdot\left(g_{\mathrm{mn} 5}+g_{\mathrm{mp} 2}\right) \cdot\left(r_{\mathrm{on} 5} \| r_{\mathrm{op} 2}\right)$.

The voltage change of the whole process is shown in Figure 9.

In order to reduce the impact of input offset voltage and kickback noise, the preamplifier is adopted, the architecture of which is shown in Figure 10. The preamplifier uses Current Starving Technical [13] to increase the gain instead of using cascode structure. The small signal gain is calculated as follows:

$$
A_{v}=\frac{g_{\mathrm{mn} 2}}{g_{\mathrm{mp} 4}}=\frac{\sqrt{2 \mu_{n} C_{\mathrm{ox}}(w / L)_{2} I_{d n 2}}}{\sqrt{2 \mu_{p} C_{\mathrm{ox}}(w / L)_{4} I_{d p 4}}}=\frac{\sqrt{\mu_{n}(w / L)_{2}}}{\sqrt{\mu_{p}(w / L)_{4}}} \cdot \frac{1}{\sqrt{1-K}} .
$$

$K$ represents the ratio coefficient of the current flowing through the $\mathrm{NM}_{4}$ or $\mathrm{NM}_{5}$, which determines the gain of amplifier. Aimed at making the resolution of latch shown in Figure 8 reach 0.5LSB, the gain of the amplifier requires at least

$$
A_{v}=\frac{V_{\text {OS,Latch }}}{1 / 2 \mathrm{LSB}}
$$

The gain is related to $V_{\text {OS,Latch }}$ closely. Based on the requirement of resolution and process characteristics, the three-stage operational amplifier is adopted.

The amplifier itself owns offset voltage, requiring the gain of the input stage maximized. The input offset voltage of the last two stages can be ignored when it is equivalent to the input. However, the offset voltages are still needed to be eliminated as much as possible, which is depicted in Figure 11.

The work of amplifier contains two phases: reset phase and amplification phase. In the reset phase, both input terminals are short to the common mode voltage $V_{\mathrm{CM}}$ through the 


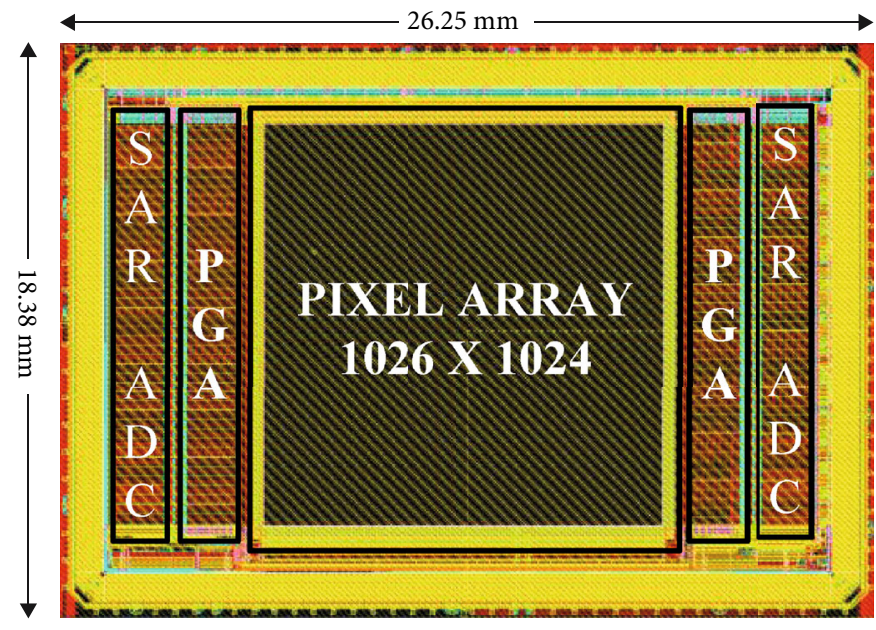

FIgURE 18: The layout of the chip.
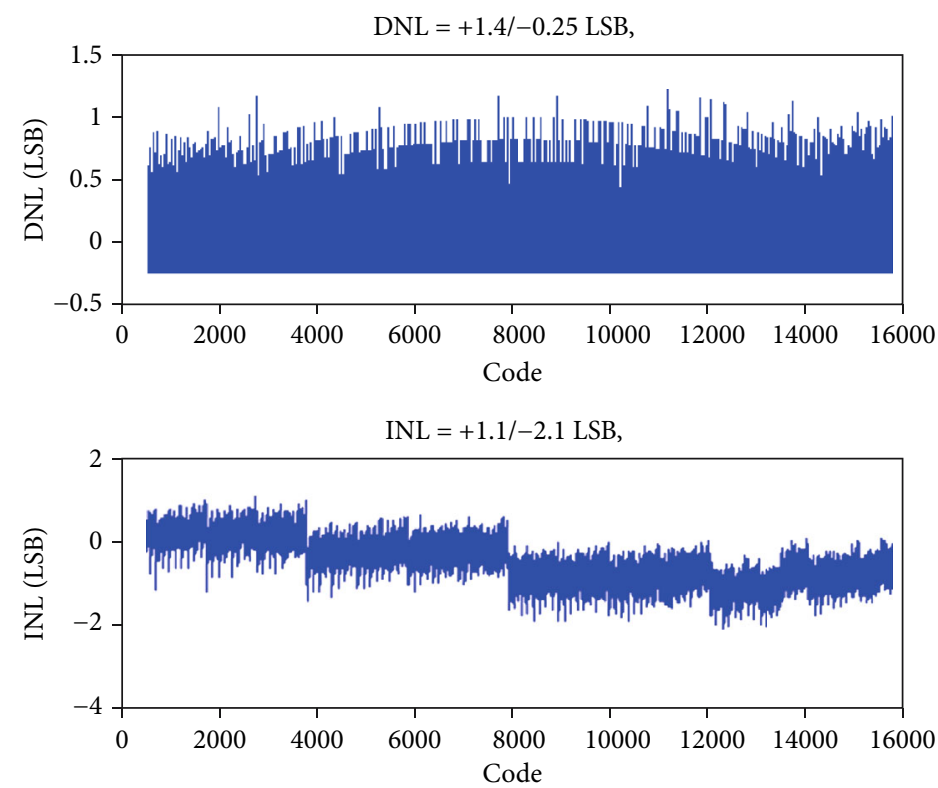

FIgURE 19: Static performance of SAR ADC.

switch when the two output terminals are also short to the $V_{\mathrm{CM}}$. The output offset is stored in capacitor $C_{1}$ and $C_{2}$, which is the opposite to the input offset:

$$
V_{\text {os,out }}=-A \cdot V_{\mathrm{os}, \text { in }} \text {. }
$$

When the amplifier enters the amplification stage, the switches connected to $V_{\mathrm{CM}}$ do not keep on and input signals $V_{\text {ip }}$ and $V_{\text {in }}$ connect to the input of amplifier. At this time, the output signal of amplifier can be obtained:

$$
V_{\text {out }}=\left(V_{\text {ip }}-V_{\text {in }}+V_{\text {os,in }}\right) \bullet A-A \bullet V_{\text {os,in }}=\left(V_{\text {ip }}-V_{\text {in }}\right) \bullet A \text {. }
$$

According to equation (30), the output does not contain $V_{\text {os }}$, which means the effect of offset voltage is eliminated.
The complete comparator design is shown in Figure 12, including amplifiers, latch, and RS flip-flop. The three-stage preamplifier amplifies the input signal, and then, the amplified signal is compared by StrongARM latch quickly. The function of RS flip-flop is to output the results to the logic register.

3.3. Reference Voltage Self-Calibration Algorithm. The proposed architecture adopts a two-step scaled reference. The resolution of two reference voltages $V_{\text {REF-TOP }}+V_{F} / 128$ and $V_{\text {REF_BOT }}+V_{F} / 128$ is up to $V_{F} / 128$, which is difficult for design. Once the accuracy of the reference voltage is far from the target value, the results of SAR ADC are greatly affected. The reference voltage self-calibration algorithm is applied to guarantee the required accuracy.

The proposed reference voltage self-calibration algorithm is based on the split capacitor linearity on-chip self- 


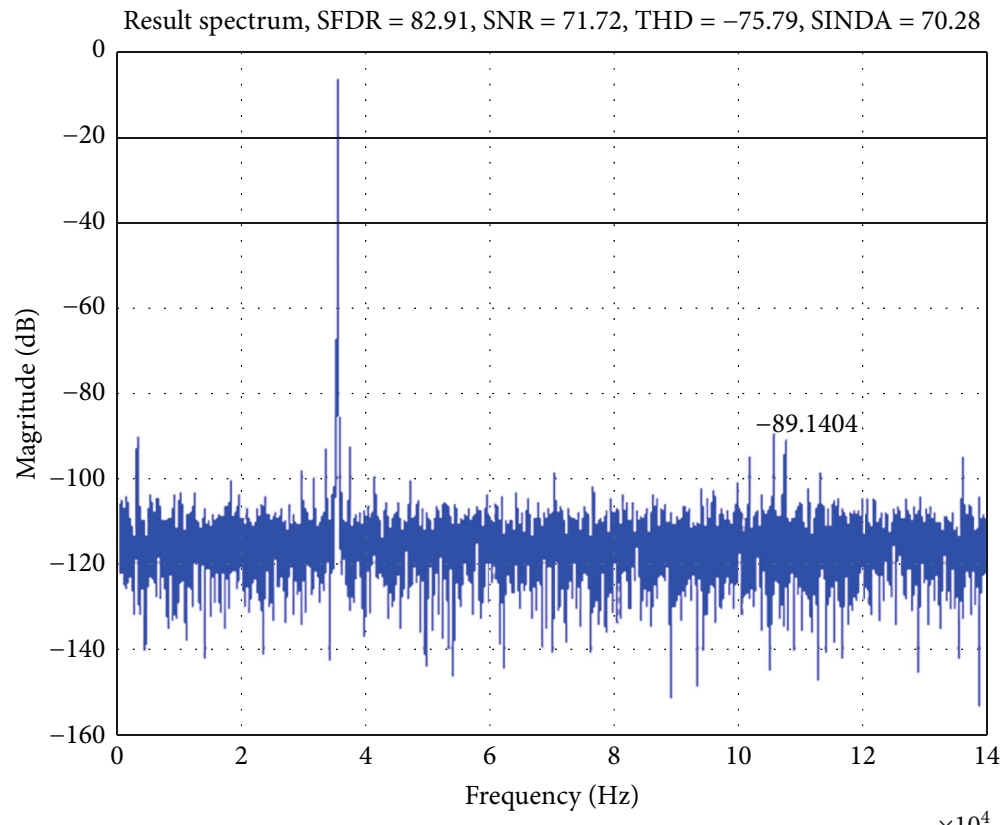

FIgURe 20: Dynamic performance of SAR ADC.

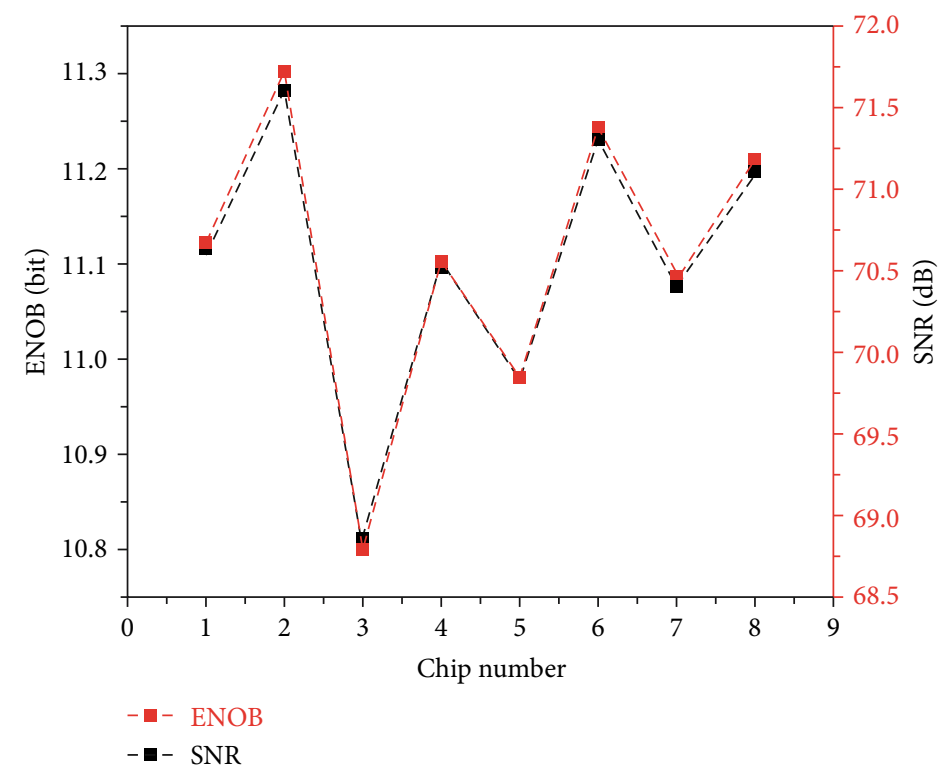

FIgURE 21: The measured SNR and ENOB of different chips.

calibration method proposed by Yoshioka et al. [14], which is to correct the output code by comparing the certain capacitors and the rest of all low capacitors. When the certain capacitance is consistent with the rest of all low capacitors, the calibration is finished.

Combining the correlated double sampling circuit technology and the above self-calibration method, the reference voltage self-calibration algorithm is proposed and the flowchart is shown in Figure 13.

The algorithm includes two patterns. Pattern 1 provides the relatively accurate target value when pattern 2 represents the value to be corrected. Two-step scaled reference is used in the process. The reference voltages $V_{\text {REF_TOP }}$ and $V_{\text {REF_BOT }}$ are the precise voltages, which generate $V_{\text {REF_TOP }}+V_{F} / 128$ or $V_{\text {REF_BOT }}+V_{F} / 128$ by DAC. The $V_{\text {REF_TOP }}+V_{F} / 128$ or $V_{\text {REF_BOT }}+V_{F} / 128$ in pattern 2 is the internally generated voltage, which is a rough value and needs to be corrected.

The precise voltages and rough voltages are converted into digital code through ADC. The difference between precise voltages and rough voltages determines the action of counter. Once the difference is equal to zero, the calibration is finished. If the difference is not equal to zero, the counter 


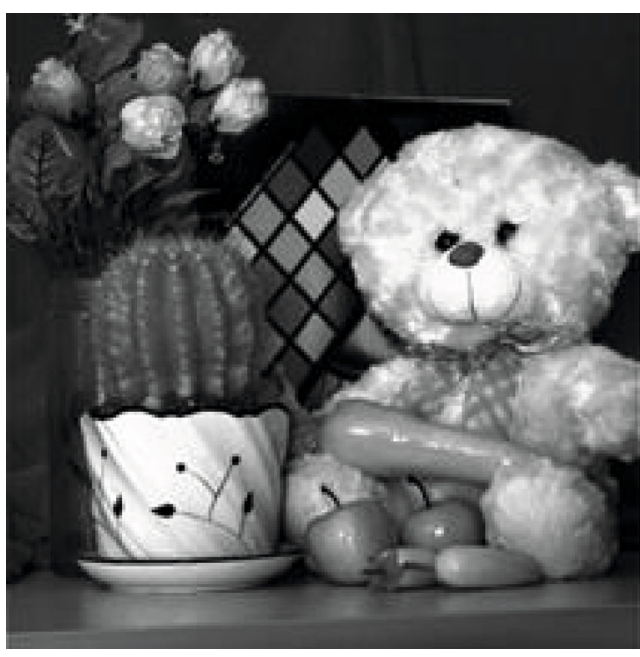

(a)

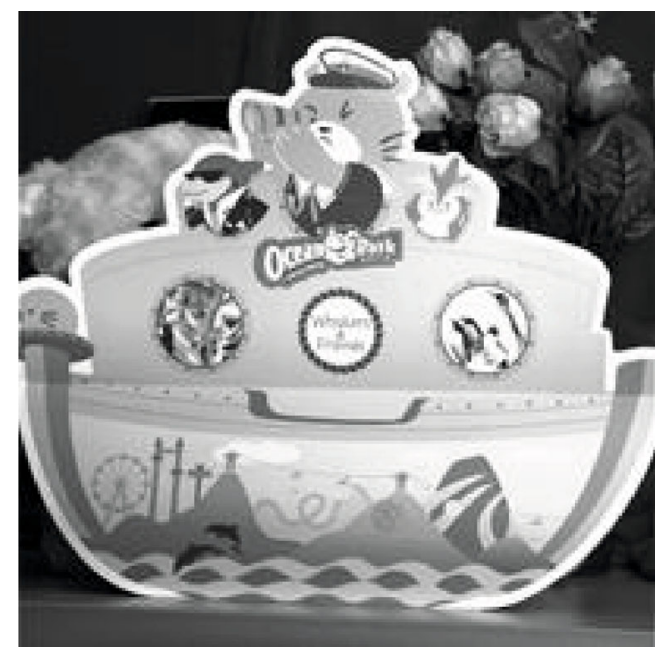

(b)

Figure 22: Captured photos by the CIS.

TABle 2: Performance summary of the prototype.

\begin{tabular}{lc}
\hline Parameter & Value \\
\hline Process & $380 \mathrm{~nm}$ CMOS process \\
Analog supply & $3.3 \mathrm{~V}$ \\
Digital supply & $3.3 \mathrm{~V}$ \\
ADC & $14 \mathrm{bits}$ \\
Resolution & $150 \mathrm{kSps}$ \\
Sample rate & $-2.4 \mathrm{~V} \sim+2.4 \mathrm{~V}$ \\
Input range & $+1.4 /-0.25 \mathrm{LSB}$ \\
DNL & $+1.1 /-2.1 \mathrm{LSB}$ \\
INL & $71.7 \mathrm{~dB}$ \\
SNR & $82.91 \mathrm{~dB}$ \\
SFDR & $70.28 \mathrm{~dB}$ \\
SINAD & $477.2 \mathrm{uW}$ \\
Power consumption & \\
PGA & $3 \mathrm{x}, 4 \mathrm{x}, 6 \mathrm{x}$ \\
Gain & \\
CIS & $50 \mathrm{fps}$ \\
Frame rate & $1026 \times 1024$ \\
Pixel number & $12.5 \mu \mathrm{m} \times 12.5 \mu \mathrm{m}$ \\
Pixel pitch & $13.6 \mathrm{e}$ rms \\
Temporal noise & $613 \mathrm{~mW}$ \\
Total power &
\end{tabular}

will add one or minus one and the output of DAC will get closer to the target value.

The capacitor array of $V_{\text {REF_BOT }}+V_{F} / 128$ calibration pattern is shown in Figure 14 when the capacitor array of $V_{\text {REF_TOP }}+V_{F} / 128$ calibration pattern is shown in Figure 15. Both work on the same principle, but the output of DAC in the $V_{\text {REF_TOP }}+V_{F} / 128$ calibration is limited. For a differential ADC, the single-ended voltage can only reach

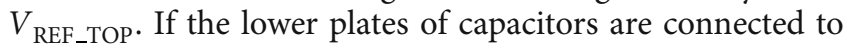

the $V_{\text {REF_TOP }}+V_{F} / 128$, the results of ADC will exceed the conversion range, which is not accurate.

Assuming that the error of A/D conversion is $\pm 1 \mathrm{LSB}$, the digital code $D_{1}$ in pattern 1 is equal to 00000010000000 when correcting $V_{\mathrm{REF} \_ \text {BOT }}+V_{F} / 128$. If the reference voltage is provided from a relatively small value by pattern 2 , the digital code will keep on the 00000010000000 , which is not consistent with the real value. However, the $D_{1}$ is equal to $D_{2}$, which means the calibration has finished. During the process, the reference voltage self-calibration algorithm does not take on the job, so the reference voltages should change from a large value and the calibration of $V_{\text {REF_TOP }}+V_{F} / 128$ is the same as well.

Figure 16 shows the timing block of calibration. PAT BUS is the bus signal that controls the DAC capacitance switch. When the system is powered on, the signal RESETD resets the digital circuit and then the signal CAL_BEGIN jumps to the high level, which means the calibration module begins to work. The timing of A/D conversion is the same as SAR ADC. A single complete calibration cycle includes 2 settings of switch mode and 2 A/D conversions. Once the second conversion is finished, the signal ADC_DONE will turn to a high level. The compared results of two conversions determine the state of calibration.

If the calibration is over, the signal CAL_OK becomes a high level, which represents the work of one reference voltage calibration has been finished. If the signal CLK_OKs of two references are high level, the whole calibration will be finished and the signal NORMAL will turn to a high level as well.

\section{Experimental Results}

The CMOS image sensor is fabricated in the $180 \mathrm{~nm}$ CMOS process, which is used in remote sensing. Referring to the capacitor array, MIM capacitors are chosen, which have a better match and less affected by temperature compared with others. The pixel array is $1026 \times 1024$, and the pixel pitch is 
TABLE 3: The comparison of previous work.

\begin{tabular}{|c|c|c|c|c|c|}
\hline Parameter & This work & JSSC 16 [15] & TED 16 [16] & ISSCC 18 [17] & JSSC 19 [18] \\
\hline Process $(\mathrm{nm})$ & 180 & 180 & 90 & 90 & 65 \\
\hline ADC type & SAR & SAR & SAR & SAR & SAR \\
\hline Supply voltage (V) & 3.3 & $2.8 / 1.8$ & $2.8 / 1.2$ & $1.8 / 1$ & 3.4 \\
\hline Number of pixel & $1026 \times 1024$ & $160 \times 120$ & $1696 \times 1212$ & $2560 \times 1536$ & $792 \times 528$ \\
\hline Pixel pitch $(\mu \mathrm{m})$ & 12.5 & 4.4 & 1.12 & 1.5 & 1.5 \\
\hline Frame rate (fps) & 50 & 100 & 60 & 60 & 170 \\
\hline Resolution (bit) & 14 & 10 & 12 & 10 & 10 \\
\hline
\end{tabular}

$12.5 \mu \mathrm{m} \times 12.5 \mu \mathrm{m}$. The photography of the chip is shown in Figure 17 and the layout of the chip is shown in Figure 18, which are consistent. The pixel array is put in the center of the chip while the readout circuits are placed on the left and right sides. The whole area of the chip is $26.25 \times 18.38$ $\mathrm{mm}^{2}$, where the pixel array occupies the main area.

When it comes to SAR ADC, the performance contains static performance and dynamic performance. Due to the combination of PGA and SAR ADC, the performance of SAR ADC is affected by PGA and the following presentation includes the effect of PGA. The static performance is shown in Figure 19. The DNL is $+1.4 /-0.25 \mathrm{LSB}$ and the INL is $+1.1 /-2.1 \mathrm{LSB}$, which reflects the transient noise. At the sampling speed of $150 \mathrm{kS} / \mathrm{s}$, the SNR and SFDR of the SAR ADC are $71.72 \mathrm{~dB}$ and $82.91 \mathrm{~dB}$, respectively, when the frequency of the input signal is $33.3 \mathrm{kHz}$, which is shown in Figure 20. What is more, the THD of SAR ADC is $-75.79 \mathrm{~dB}$ and the SINAD is $70.28 \mathrm{~dB}$. In order to verify the stability performance of the proposed prototype, eight chips are tested to get the data, which is presented in Figure 21. The SNR of SAR ADC is from $68.78 \mathrm{~dB}$ to $71.71 \mathrm{~dB}$ when the ENOB is from 10.8 bits to 11.3 bits, whose performance is relatively stable. The single SAR ADC consumes $477.2 \mathrm{uW}$.

Figure 22 shows the photos captured by the proposed CIS chips, in which the edge of the subjects can be clearly recognized. What is more, the depth of the background color can be clearly identified, which means the CIS have a good resolution. The performance summary of the prototype is listed in Table 2, including SAR ADC, PGA, and the CIS. The total power consumption of the CIS is about $613 \mathrm{~mW}$.

Compared with the previous works, which are depicted in Table 3, this work adopts the large pixel array and the resolution of SAR ADC is comparatively high without the help of complex calibration and the advanced technology. The proposed CIS chip keeps a balance between the area, resolution, and speed.

\section{Conclusions}

The 14-bit fully differential SAR ADC with PGA is proposed to apply on CIS. In this paper, the scale of pixel array is large when the three-way PGA is used to sample at the same time to increase the speed. What is more, it also provides three types of gain: $3 \mathrm{x}, 4 \mathrm{x}$, and $6 \mathrm{x}$. Considering the reset and exposure signal, the fixed deviation is added into the PGA, which makes the input signal distributed evenly on the positive and negative sides. When it comes to SAR ADC, the two-step scaled-reference voltages are adopted to realize the goal of 14-bit A/D conversion with a 7-bit complementary capacitor array, which is aimed at reducing the number and the area of capacitors. In order to make the precision of reference voltage meet the requirement, the reference voltage selfcalibration algorithm is used. During the whole process, the offset and matching accuracy needs to be considered as well. By finishing the above design, the readout circuit realizes the function well and the proposed CIS achieves the goal of high resolution for remote sensing, which are verified in the manufactured chips.

\section{Data Availability}

The data used to support the findings of this study are available from the corresponding author upon request.

\section{Conflicts of Interest}

The authors declare that they have no conflicts of interest.

\section{Acknowledgments}

This work was supported by the National Key R\&D Program of China (2018YFB0904900 and 2018YFB0904902).

\section{References}

[1] J. Wang, S. Liu, Y. Shen, and Z. Zhu, "Low-power single-ended SAR ADC using symmetrical DAC switching for image sensors with passive CDS and PGA technique," IEEE Transactions on Circuits and Systems I: Regular Papers, vol. 65, no. 8, pp. 2378-2388, 2018.

[2] S. I. Hwang, J. H. Chung, H. J. Kim et al., “A 2.7-M pixels 64$\mathrm{mW}$ CMOS image sensor with multicolumn-parallel noiseshaping SAR ADCs," IEEE Transactions on Electron Devices, vol. 65 , no. 3, pp. 1119-1126, 2018.

[3] J. Lee, H. Park, B. Song et al., "High frame-rate VGA CMOS image sensor using non-memory capacitor two-step singleslope ADCs," IEEE Transactions on Circuits and Systems I: Regular Papers, vol. 62, no. 9, pp. 2147-2155, 2015.

[4] S. Xie and A. Theuwissen, "A 10 bit 5 MS/s column SAR ADC with digital error correction for CMOS image sensors," IEEE Transactions on Circuits and Systems II: Express Briefs, vol. 67, no. 6, pp. 984-988, 2020. 
[5] R. Kuroda, Y. Tochigi, K. Miyauchi et al., “A 20Mfps global shutter CMOS image sensor with improved light sensitivity and power consumption performances," ITE Transactions on Media Technology and Applications, vol. 4, no. 2, pp. 149154, 2016.

[6] D. G. Chen, F. Tang, M. K. Law, and A. Bermak, "A 12 pj/pixel analog-to-information converter based $816 \times 640$ pixel cmos image sensor," IEEE Journal of Solid-State Circuits, vol. 49, no. 5, pp. 1210-1222, 2014.

[7] X. Zhang, W. Fan, J. Xi, and L. He, "A 14-bit 150KS/s SAR ADC with PGA for CMOS image sensor," in 2019 IEEE International Symposium on Signal Processing and Information Technology (ISSPIT), pp. 1-5, Ajman, United Arab Emirates, 2019.

[8] R. Hogervorst, J. P. Tero, R. G. H. Eschauzier, and J. H. Huijsing, "A compact power-efficient $3 \mathrm{~V}$ CMOS rail-to-rail input/output operational amplifier for VLSI cell libraries," IEEE Journal of Solid-State Circuits, vol. 29, no. 12, pp. 1505-1513, 1994.

[9] R. S. Assaad and J. Silva-Martinez, "The recycling folded cascode: a general enhancement of the folded cascode amplifier," IEEE Journal of Solid-State Circuits, vol. 44, no. 9, pp. 25352542, 2009.

[10] J. L. McCreary and P. R. Gray, "All-MOS charge redistribution analog-to-digital conversion techniques. I," IEEE Journal of Solid-State Circuits, vol. 10, no. 6, pp. 371-379, 1975.

[11] M.-S. Shin and O.-K. Kwon, "14-bit two-step successive approximation ADC with calibration circuit for highresolution CMOS imagers," Electronics Letters, vol. 47, no. 14, pp. 790-791, 2011.

[12] B. Razavi, "The StrongARM latch [a circuit for all seasons]," IEEE Solid-State Circuits Magazine, vol. 7, no. 2, pp. 12-17, 2015.

[13] L. Yao, M. S. J. Steyaert, and W. Sansen, “A 1-V 140-mu W 88$\mathrm{dB}$ audio sigma-delta modulator in 90-nm CMOS," Journal of Solid-State Circuits, vol. 39, no. 11, pp. 1810-1812, 2004.

[14] M. Yoshioka, K. Ishikawa, T. Takayama, and S. Tsukamoto, “A 10-b 50-MS/s 820- $\mu \mathrm{W}$ SAR ADC with on-chip digital calibration," IEEE transactions on biomedical circuits and systems, vol. 4, no. 6, pp. 410-416, 2010.

[15] H. J. Kim, S. I. Hwang, J. W. Kwon et al., "A delta-readout scheme for low-power CMOS image sensors with multicolumn-parallel SAR ADCs," IEEE Journal of Solid-State Circuits, vol. 51, no. 10, pp. 2262-2273, 2016.

[16] M. K. Kim, S. K. Hong, and O. K. Kwon, "An area-efficient and low-power 12-b SAR/single-slope ADC without calibration method for CMOS image sensors," IEEE Transactions on Electron Devices, vol. 63, no. 9, pp. 3599-3604, 2016.

[17] O. Kumagai, A. Niwa, K. Hanzawa et al., "A 1/4-inch 3.9 Mpixel low-power event-driven back-illuminated stacked CMOS image sensor," in 2018 IEEE International Solid - State Circuits Conference - (ISSCC), pp. 86-88, San Francisco, CA, 2018.

[18] K. D. Choo, L. Xu, Y. Kim et al., "Energy-efficient motiontriggered IoT CMOS image sensor with capacitor arrayassisted charge-injection SAR ADC," IEEE Journal of SolidState Circuits, vol. 54, no. 11, pp. 2921-2931, 2019. 